\title{
Computer-aided design of nanocapsules for therapeutic delivery
}

\author{
Zeina Shreif and Peter Ortoleva* \\ Center for Cell and Virus Theory, Department of Chemistry, Indiana University, Bloomington, \\ IN 47405, USA
}

(Received 21 December 2007; final version received 22 April 2008)

\begin{abstract}
The design of nanocapsules for targeted delivery of therapeutics presents many, often seemingly self-contradictory, constraints. An algorithm for predicting the physicochemical characteristics of nanocapsule delivery and payload release using a novel allatom, multiscale technique is presented. This computational method preserves key atomic-scale behaviours needed to make predictions of interactions of functionalized nanocapsules with the cell surface receptors, drug, siRNA, gene or other payload. We show how to introduce a variety of order parameters with distinct character to enable a multiscale analysis of a complex system. The all-atom formulation allows for the use of an interatomic force field, making the approach universal and avoiding recalibration with each new application. Alternatively, key parameters, which minimize the need for calibration, are also identified. Simultaneously, the methodology enables predictions of the supra-nanometer-scale behaviour, such as structural transitions and disassembly of the nanocapsule accompanying timed payload release or due to premature degradation. The final result is a Fokker-Planck equation governing the rate of stochastic payload release and structural changes and migration accompanying it. A novel "salt shaker" effect that underlies fluctuation-enhancement of payload delivery is presented. Prospects for computer-aided design of nanocapsule delivery system are discussed.
\end{abstract}

Keywords: nanomedicine; nanocapsules; liposomes; drug delivery; computer-aided design

\section{Background}

The delivery of cancer drugs, siRNA or genes via a functionalized nanocapsule is a subject of great interest. Current methods for treating cancer are generally intrusive, involving long and repeated procedures (surgery, chemotherapy and radiation). In addition, chemotherapeutics have strong side effects so that they cannot be administered in sufficiently high doses to kill all the abnormal cells without affecting the healthy ones [1]. Hence, the need for drugs that have the ability to target only the cancer cells without affecting other tissues is a current objective, which, however, may not be feasible. In other words, a way to deliver the drug only when it reaches the surface of the tumour is needed [2-4]. Another major requirement for drug delivery nanocapsules is the ability to control the release of the drug over a long period of time with constant concentration [3]. Other promising applications of nanocapsule delivery are for siRNA [5-7] and genes [8-10] therapies.

An example of well-studied drug delivery systems is liposome-based nanocapsules $[2,11-25,38,39]$. Because of the broad range of size, shape, surface morphology,

*Corresponding author. Email: ortoleva@indiana.edu

ISSN 1748-670X print/ISSN 1748-6718 online 
composition, surface charge and bilayer fluidity [11] in which they can be created, liposomes constitute a very promising solution to the challenges encountered during drug delivery. For efficient anti-tumour delivery, liposomes should be small enough to avoid MPS uptake and thus reduce toxicity and prolong circulation in the blood, and big enough to be able to selectively enter tumour pores taking advantage of the tumour's increased permeability compared to normal tissues. Altering lipid composition affects not only the affinity of the liposomal carrier to certain tissues, but also its circulation time and rate of release of the drug encapsulated in it; while saturated phospholipids reduce membrane fluidity and thus prolong circulation in the blood, negatively charged lipids have the opposite effect [12]. Similarly, lipids with low phase transition temperature (short, unsaturated fatty acid chains) increase rate of drug release while those with high phase transition (long, unsaturated fatty acid chains) decrease release rate [13]. It was also discovered that circulation time can be greatly enhanced by coating the liposomes with polymer polyethylene glycol, PEG (Stealth liposomes) [14]. However, this method was found to cause additional side effects such as skin toxicity [15]. Finally, it is important that drug is released at the surface of the target cell. This can be done either by coating the liposome with antibodies - immunoliposomes - $[16,17]$ or attaching ligands that target specific receptors at the site of interest [18]; or by developing liposomes that are sensitive to specific triggers such as $\mathrm{pH}$ [19-21], heat [22,23], light [24] or enzyme [25].

Considering the variations in the nature of the payload and the thermal and chemical environments that nanocapsules must address, it is necessary to have a general physicochemical simulator that can be used in computer-aided nanocapsule therapeutic delivery. Prediction of the rate of drug release for given nanocapsule structure and conditions in the microenvironment based on a parameter-free model of supra-molecular structures that would optimize payload targeting would be a valuable asset.

Pharmacodynamics/pharmacokinetics models have been built in order to study the optimum release scenario to achieve high anti-tumour activity [26]. Other theoretical models have been developed to simulate drug release from polymeric delivery systems (see Ref. [27] for a good review). These models are either empirical or mechanistic. Empirical models $[28,29]$ only take into consideration the overall order of the rate of drug release while mechanistic ones [30,31] take into account the mechanisms involved in the rate-limiting step such as diffusion, swelling and erosion. However, these models are macroscopic and do not take into consideration atomistic effects, and therefore neglect fluctuations.

Release of payloads takes seconds to hours, a timescale not accessible to existing molecular dynamics codes, which are impractical for suprananosecond studies. Computations are further hindered because of the millions of atoms that must be accounted for in a nanometer-scale problem that involves the payload, capsule and microenvironment. While payload release is a suppressed timescale process, atomic collisions/vibrations take place on the $10^{-12}$ second scale. The objective of this study is to show how such systems can be simulated even though they support phenomena that simultaneously involve many scales across space and time. An all-atom multiscale approach has been developed recently for simulating the migration and structural transitions of nanoparticles and other nanoscale phenomena [32-35], and is applied here to the nanocapsule delivery problem.

The starting point of our approach is the identification of variables (order parameters) that capture the nanoscale features of the payload/capsule/host system, can be demonstrated to be slow via Newton's equations, and are "complete" (i.e. there are no other slow variables that couple to them). Examples of these order parameters are 
nanocapsule centre of mass $(\mathrm{CM})$ position, orientation and similar measures of the payload and other objects in the microenvironment. We show how a variety of types of order parameters needed to describe specific nanoscale features can be integrated into a fully coupled model of a complex bionanosystem. Next, we formulate the Liouville equation that yields the statistical dynamics of the positions and momenta of all atoms in the system and, via multiscale techniques [33-35], the order parameters as well. A perturbation technique is used to derive a Fokker-Planck (FP) equation for the dynamics of the order parameters alone. While multiscale analysis has a long history of application to Brownian motion (see Refs. $[32,33]$ for reviews), our approach introduces novel technical advances that capture key aspects of the nanoscale structures needed for nanocapsule therapeutic delivery analysis.

There are several strategies for targeted delivery and associated phenomena that should be incorporated in a simulation approach. Like viruses that target specific types of host cells, the nanocapsule could be constructed of proteins whose sequence and structure allow binding to selected cell surface protein/receptor sites. Alternatively, injected nanocapsules could contain a magnetic component part [36] that allows the use of external applied fields to guide them to target tissue. Finally, release of the payload must be correctly controlled spatially and temporally, by either designing nanocapsule disassembly to initiate upon contact with or emersion into the cell membrane, or manipulating it by application of localized heating in the target area. The simulation algorithm must account for the migration of a nanostructure and its structural stability, i.e. to predict thermal or host medium conditions favouring a leaky structure or disassembly of the nanocapsule in the target medium. In summary, we present a mathematical approach that can be used for the computer-aided design of nanocapsule therapeutic payload delivery systems.

In this study, we formulate the nanocapsule delivery problem in terms of a set of order parameters characterizing the transporter capsule and its payload (Section II). We use multiscale analysis to derive an FP equation for this set of interacting order parameters (Section III). We present predicted drug release scenarios and show that calibration can be reduced to two parameters, friction and barrier height. Given the size and properties of both nanocapsule and its payload, the rate of release can be predicted. Alternatively, given a release rate and calibrated friction coefficients, the optimum size of the nanocapsule can be found (Section IV). We discuss the notion of fluctuation enhanced payload release (Section V) and draw conclusions in Section VI.

\section{Order parameters for migration, structural transformation and dispersal}

The nanocapsule/payload delivery system displays several distinct behavioural regimes. These include long-length scale migration to and across the diseased tissue, random motion in the vicinity of target cell surfaces, interaction with these surfaces and changes induced by variations in temperature or chemical environment that initiate and sustain drug release. In the present study, we focus on the short-scale processes and not the long-scale transport to the target tissue. For simplicity, we do not consider the cell surface explicitly; rather, we focus on payload release within a host fluid. However, our methodology can also be applied to a wider range of phenomena.

The system of interest consists of the nanocapsule, payload and host medium. It is described in terms of its $N$ classical atoms, which interact via bonded and non-bonded forces. The all-atom multiscale approach starts with the identification of order parameters and a reformulation of the Liouville equation. We arrive at an FP equation for stochastic order parameter dynamics. We follow our earlier methodology developed for other problems [33-35,37], except we introduce several technical innovations. 
Denote the CM position of the nanocapsule $\vec{R}$ and that of the drug or other payload $\vec{R}_{\mathrm{d}}$ :

$$
\begin{aligned}
\vec{R} & =\sum_{i=1}^{N} \frac{m_{i} \vec{r}_{i}}{m^{*}} \Theta_{i} \\
\vec{R}_{\mathrm{d}} & =\sum_{i=1}^{N} \frac{m_{i} \vec{r}_{i}}{m_{\mathrm{d}}^{*}} \Theta_{i}^{\mathrm{d}},
\end{aligned}
$$

where $m_{i}$ and $\vec{r}_{i}$ are the mass and position of the $i$ th atom; $m^{*}=\sum_{i=1}^{N} m_{i} \Theta_{i}$, and $m_{\mathrm{d}}^{*}=$ $\sum_{i=1}^{N} m_{i} \Theta_{i}^{\mathrm{d}}$ are the total mass of the nanocapsule and payload. $\Theta_{i}=1$ when $i$ is in the nanocapsule and zero otherwise; and similarly with $\Theta_{i}^{\mathrm{d}}$ for the payload. If the CM coordinates are to be used as order parameters, one should first demonstrate that they evolve slowly. Newton's equations imply $\mathrm{d} \vec{R} / \mathrm{d} t=-L \vec{R}$ and $\mathrm{d} \vec{R}_{\mathrm{d}} / \mathrm{d} t=-L \vec{R}_{\mathrm{d}}$, where $L$ is the Liouville operator:

$$
L=-\sum_{i=1}^{N}\left[\frac{\vec{p}_{i}}{m_{i}} \cdot \frac{\partial}{\partial \vec{r}_{i}}+\vec{F}_{i} \cdot \frac{\partial}{\partial \vec{p}_{i}}\right]
$$

where $\vec{F}_{i}$ and $\vec{p}_{i}$ are the force on and momentum of atom $i$. Introducing the total momentum of the nanocapsule $\vec{P}^{*}$ and that of the payload $\vec{P}_{\mathrm{d}}^{*}$ yields $\mathrm{d} \vec{R} / \mathrm{d} t=\vec{P} * / m^{*}$ and $\mathrm{d} \vec{R}_{\mathrm{d}} / \mathrm{d} t=\vec{P}_{\mathrm{d}}^{*} / m_{\mathrm{d}}^{*}$, where $\vec{P}^{*}=\sum_{i=1}^{N} \vec{p}_{i} \Theta_{i}$ and $\vec{P}_{\mathrm{d}}^{*}=\sum_{i=1}^{N} \vec{p}_{i} \Theta_{i}^{\mathrm{d}}$. Similarly, $\mathrm{d} \vec{P} * / \mathrm{d} t=$ $-L \vec{P} *=\sum_{i=1}^{N} \vec{F}_{i} \Theta_{i}$ (the net force on the nanocapsule) and $\mathrm{d} \vec{P}_{\mathrm{d}}^{*} / \mathrm{d} t=-L \vec{P}_{\mathrm{d}}^{*}=\sum_{i=1}^{N} \vec{F}_{i} \Theta_{i}^{\mathrm{d}}$ (the net force on the payload). This completes Newton's equations for the CM variables for the nanocapsule and payload.

Both payload and nanocapsule are composed of many atoms (typically millions); thus, we take them to have a diameter of $O\left(\varepsilon^{-1}\right)$ for small factor $\varepsilon$; while $\vec{R}$ and $\vec{R}_{\mathrm{d}}$ are scaled as $O\left(\varepsilon^{0}\right)$. To define $\varepsilon$, we take $\varepsilon^{2}=m / m *$ for typical mass of a capsule atom $m$, and similarly $\varepsilon^{2}=m_{\mathrm{d}} / m_{\mathrm{d}}^{*}$, where $m_{\mathrm{d}} \equiv m m_{\mathrm{d}}^{*} / m^{*}$ and is on the order of the mass of a typical payload atom. The $\varepsilon^{2}$ scaling is consistent with the fact that the nanocapsule is a shell-like object and the payload fits inside the nanocapsule initially.

Under the assumption that the system is near equilibrium with respect to the momentum degrees of freedom, the CM momentum of the nanocapsule scales as the square root of its mass, i.e. $P^{* 2} / m * \sim k_{\mathrm{B}} T$. Thus, $\vec{P} *$ is $O\left(\varepsilon^{-1}\right)$, and similarly for $\vec{P}_{\mathrm{d}}^{*}$. With this, we adopt the scaled variables $\vec{P}$ and $\vec{P}_{\mathrm{d}}$ such that

$$
\vec{P}^{*}=\varepsilon^{-1} \vec{P}, \quad \vec{P}_{\mathrm{d}}^{*}=\varepsilon^{-1} \vec{P}_{\mathrm{d}}
$$

Near equilibrium there is much cancellation of the individual forces acting on a nanostructure. Although there are $O\left(\varepsilon^{-2}\right)$ atoms in the nanocapsule, partial cancellation is assumed to make a net force that scales as $O\left(\varepsilon^{0}\right)$, not $O\left(\varepsilon^{-2}\right)$, i.e. not as the surface area of the nanocapsule. Thus, we introduce net forces via $\vec{f}=\sum_{i=1}^{N} \vec{F}_{i} \Theta_{i}$ for the nanocapsule and $\vec{f}_{\mathrm{d}}=\sum_{i=1}^{N} \vec{F}_{i} \Theta_{i}^{\mathrm{d}}$ for the payload. In summary, Newton's equations imply

$$
\begin{gathered}
\frac{\mathrm{d} \vec{R}}{\mathrm{~d} t}=\varepsilon \frac{\vec{P}}{m}, \quad \frac{\mathrm{d} \vec{R}_{\mathrm{d}}}{\mathrm{d} t}=\varepsilon \frac{\vec{P}_{\mathrm{d}}}{m_{\mathrm{d}}}, \\
\frac{\mathrm{d} \vec{P}}{\mathrm{~d} t}=\varepsilon \vec{f}, \quad \frac{\mathrm{d} \vec{P}_{\mathrm{d}}}{\mathrm{d} t}=\varepsilon \vec{f}_{\mathrm{d}} .
\end{gathered}
$$


We conclude that $\vec{R}, \vec{R}_{\mathrm{d}}, \vec{P}$ and $\vec{P}_{\mathrm{d}}$ are slowly varying, qualifying them as order parameters. Furthermore, they comprise a self-consistent set in that they all evolve on the same timescale, i.e. $O\left(\varepsilon^{-1}\right)$.

To describe payload release, we introduce additional variables characterizing structural transitions in the nanocapsule (with attendant permeability changes) and dispersal (i.e. spatial extent of the cloud of payload molecules). For the former, order parameters $\Phi$ and rotation matrix $\vec{X}$ provide measures of capsule dilatation and orientation:

$$
\Phi=\sum_{i=1}^{N} \frac{m_{i} \vec{s}_{i} \cdot \overrightarrow{\bar{X}} \hat{s}_{i}^{0} \Theta_{i}}{m^{*}},
$$

where $\vec{s}_{i}$ is the position of atom $i$ relative to $\vec{R} ; \overrightarrow{\bar{X}}$ is a length-preserving rotation matrix that depends on a set of three Euler angles specifying nanocapsule orientation; $\hat{s}_{i}^{0}=\vec{s}_{i}^{0} / s_{i}^{0}$, where $s_{i}^{0}$ is the length of $\vec{s}_{i}^{0}$ and the superscript 0 indicates a reference nanocapsule structure. Note that $\vec{S}_{i}^{0}$ is not a dynamical variable but, for example, is derived from cryoTEM data. As $\Phi$ is a sum of $O\left(\varepsilon^{-2}\right)$ terms, each of $O\left(\varepsilon^{2}\right), \Phi$ is $O\left(\varepsilon^{0}\right)$.

Newton's equations imply

$$
\frac{\mathrm{d} \Phi}{\mathrm{d} t}=-L \Phi=\frac{1}{m^{*}} \sum_{i=1}^{N}\left(\frac{\vec{p}_{i}}{m_{i}}-\frac{\vec{P} *}{m^{*}}\right) \cdot \overrightarrow{\bar{X}} \hat{s}_{i}^{0} m_{i} \Theta_{i}
$$

An additional term from $\mathrm{d} \overrightarrow{\bar{X}} / \mathrm{d} t$ has been neglected for simplicity; it is small relative to $\mathrm{d} \vec{s}_{i} / \mathrm{d} t$ due to the large moment of inertia of the nanocapsule (see Ref. [34]). Define $\Pi *$ such that

$$
\Pi *=\sum_{i=1}^{N} \vec{\pi}_{i} \cdot \overrightarrow{\bar{X}} \hat{s}_{i}^{0} \Theta_{i}, \quad \frac{\vec{\pi}_{i}}{m_{i}}=\frac{\vec{p}_{i}}{m_{i}}-\frac{\vec{P}^{*}}{m^{*}} .
$$

The contribution to $\Pi^{*}$ of $O\left(\varepsilon^{-2}\right)$ terms of fluctuating signs; hence $\Pi^{*}$ scales as the square root of the number of contributing terms. Thus, we introduce the scaled parameter $\Pi=\varepsilon \Pi^{*}$. With this, $\Phi$ and $\Pi$ evolve via

$$
\frac{\mathrm{d} \Phi}{\mathrm{d} t}=\varepsilon \frac{\Pi}{m},
$$

And to good approximation

$$
\frac{\mathrm{d} \Pi}{\mathrm{d} t}=\varepsilon g, \quad g=\sum_{i=1}^{N} \vec{F}_{i} \cdot \overrightarrow{\bar{X}} \hat{s}_{i}^{0} \Theta_{i}
$$

for "dilatation force" $g$ that we take to scale as $\varepsilon^{0}$ using an argument similar to that for $\vec{f}$ and $\vec{f}_{\mathrm{d}}$. Thus, $\Phi$ and $\Pi$ are slowly varying and are a consistent set of order parameters.

An order parameter $\Lambda$ is introduced to describe the dispersal of the payload:

$$
\Lambda=\sum_{i=1}^{N} m_{i} s_{i}^{\mathrm{d}} \Theta_{i}^{\mathrm{d}} / m_{\mathrm{d}}^{*}
$$

where $\vec{s}_{i}^{\mathrm{d}}$ is the position of atom $i$ relative to $\vec{R}_{\mathrm{d}}$ and $s_{i}^{\mathrm{d}}$ is its length. As argued for $\Phi, \Lambda$ scales as $O\left(\varepsilon^{0}\right)$. 
Newton's equations imply

$$
\frac{\mathrm{d} \Lambda}{\mathrm{d} t}=\sum_{i=1}^{N} \frac{m_{i}}{m_{\mathrm{d}}^{*}}\left[\frac{\vec{p}_{i}}{m_{i}}-\frac{\vec{P}_{\mathrm{d}}^{*}}{m_{\mathrm{d}}^{*}}\right] \cdot \hat{s}_{i}^{\mathrm{d}} \Theta_{i}^{\mathrm{d}}
$$

where $\hat{s}_{i}^{\mathrm{d}}=\vec{s}_{i}^{\mathrm{d}} / s_{i}^{\mathrm{d}}$. Introducing relative velocities $\left(\vec{\pi}_{i}^{\mathrm{d}} / m_{i}\right)=\left(\vec{p}_{i} / m_{i}\right)-\left(\vec{P}_{\mathrm{d}}^{*} / m_{\mathrm{d}}^{*}\right)$ for payload atoms yields

$$
\frac{\mathrm{d} \Lambda}{\mathrm{d} t}=\frac{\varepsilon \Pi_{\mathrm{d}}}{m_{\mathrm{d}}}, \quad \Pi_{\mathrm{d}}=\varepsilon \sum_{i=1}^{N} \vec{\pi}_{i}^{\mathrm{d}} \cdot \hat{s}_{i}^{\mathrm{d}} \Theta_{i}^{\mathrm{d}}
$$

The scaling of $\Pi_{\mathrm{d}}$ is based on the assumption that while there are $O\left(\varepsilon^{-2}\right)$ atoms in the payload, the contributions to the sum in Equation (14) are of fluctuating sign, and thus $\sum_{i=1}^{N} \vec{\pi}_{i}^{\mathrm{d}} \cdot \hat{s}_{i}^{\mathrm{d}} \Theta_{i}^{\mathrm{d}}$ is $O\left(\varepsilon^{-1}\right)$, a result again consistent with the scaling of $\vec{P}_{\mathrm{d}}^{*}$ and which reflects the near-equilibrium state of the momentum degrees of freedom. With this

$$
\frac{\mathrm{d} \Pi_{\mathrm{d}}}{\mathrm{d} t}=-L \Pi_{\mathrm{d}}=\varepsilon \sum_{i=1}^{N}\left\{\vec{F}_{i} \cdot\left(\hat{s}_{i}^{\mathrm{d}}-\sum_{j=1}^{N} \frac{m_{j} \hat{s}_{j}^{\mathrm{d}}}{m_{\mathrm{d}}^{*}} \Theta_{j}^{\mathrm{d}}\right)+\frac{\left[\left(\pi_{i}^{\mathrm{d}}\right)^{2}-\left(\vec{\pi}_{i}^{\mathrm{d}} \cdot \hat{s}_{i}^{\mathrm{d}}\right)^{2}\right]}{m_{i} s_{i}^{\mathrm{d}}}\right\} \Theta_{i}^{\mathrm{d}} .
$$

The $j$-sum is over many vector contributions, which tend to cancel; as the $m_{\mathrm{d}}^{*}$ factor in this term is proportional to the number of atoms in the payload, the $j$-sum acts like an average and therefore is small relative to the unit vector $\hat{s}_{i}^{\mathrm{d}}$. Similarly, the second term is $O(\varepsilon)$ and is therefore negligible. Thus, to good approximation

$$
\frac{\mathrm{d} \Pi_{\mathrm{d}}}{\mathrm{d} t}=\varepsilon h, \quad h=\sum_{i=1}^{N} \vec{F}_{i} \cdot \hat{s}_{i}^{\mathrm{d}} \Theta_{i}^{\mathrm{d}}
$$

for "dispersal force" $h$. We conclude that $\Lambda$ and $\Pi_{\mathrm{d}}$ constitute a self-consistent set of order parameters.

It can be demonstrated that $\mathrm{d} \overrightarrow{\vec{X}} / \mathrm{d} t$ is $O(\varepsilon)$ so the rotation is slow [33]. In what follows, we make the simplifying assumption that the coupling of dilatation or dispersal with overall slow capsule rotation can be neglected, an assumption warranting further investigation in the future. For example, for capsules whose properties are far from spherically symmetric (see Figure 1), payload release may be enhanced by, and in the same time, propel rotation.

The set of order parameters and associated momenta introduced above constitute the starting point for the multiscale analysis of the nanocapsule/payload/host medium system presented in this study. We suggest that this set constitutes a minimal description capturing many nanocapsule delivery phenomena.

\section{Equations of stochastic therapeutic delivery}

Multiscale approach is now used to derive an FP equation of stochastic dynamics for the order parameters of Section II. We follow the prescription of Refs. [33-35,37]. For the nanocapsule/payload delivery problem, the $N$-atom probability density $\rho$ is taken to have the dependence

$$
\rho\left(\Gamma ; \mathrm{Z}, \mathrm{Y}, t_{0}, \underline{t}\right)
$$






Figure 1. Dispersal can be enhanced when the capsule is spinning and escape is local; conversely, local asymmetric dispersal can drive spinning.

with $\Gamma \equiv\left\{\Gamma_{\mathrm{r}}, \Gamma_{\mathrm{p}}\right\}, \Gamma_{\mathrm{r}}=\left\{\vec{r}_{1}, \ldots, \vec{r}_{N}\right\}$ and $\Gamma_{\mathrm{p}}=\left\{\vec{p}_{1}, \ldots, \vec{p}_{N}\right\} ; Z \equiv\left\{\vec{R}, \vec{R}_{\mathrm{d}}, \Phi, \Lambda\right\}$ and $\Upsilon \equiv\left\{\vec{P}, \vec{P}_{\mathrm{d}}, \Pi, \Pi_{\mathrm{d}}\right\}$; for simplicity $\vec{X}$ and its rate of change $\vec{\Omega}$ are not accounted for explicitly. A set of scaled times, $t_{n}=\varepsilon^{n} t,(n=0,1, \ldots)$, is introduced to capture the various ways in which $\rho$ depends on time. Through the ansatz Equation (17), we account for the multiple ways in which $\rho$ depends on $\Gamma$, i.e. both directly, and through $Z$ and $Y$, indirectly. Finally, $\underline{t}=\left\{t_{1}, t_{2}, \ldots\right\}$ represents the set of long-time variables.

With this and the chain rule, the Liouville equation takes the form

$$
\begin{gathered}
\sum_{n=0}^{\infty} \varepsilon^{n} \frac{\partial \rho}{\partial t_{n}}=\left(L_{0}+\varepsilon L_{1}\right) \rho, \\
L_{0}=-\sum_{i=1}^{N}\left[\frac{\vec{p}_{i}}{m_{i}} \cdot \frac{\partial}{\partial \vec{r}_{i}}+\vec{F}_{i} \cdot \frac{\partial}{\partial \vec{p}_{i}}\right], \\
L_{1}=-\left[\frac{\vec{P}}{m} \cdot \frac{\partial}{\partial \vec{R}}+\vec{f} \cdot \frac{\partial}{\partial \vec{P}}+\frac{\Pi}{m} \frac{\partial}{\partial \Phi}+g \frac{\partial}{\partial \Pi}+\frac{\vec{P}_{\mathrm{d}}}{m_{\mathrm{d}}} \cdot \frac{\partial}{\partial \vec{R}_{\mathrm{d}}}+\vec{f}_{\mathrm{d}} \cdot \frac{\partial}{\partial \vec{P}_{\mathrm{d}}}+\frac{\Pi_{\mathrm{d}}}{m_{\mathrm{d}}} \frac{\partial}{\partial \Lambda}+h \frac{\partial}{\partial \Pi_{\mathrm{d}}}\right] .
\end{gathered}
$$

From the chain rule, derivatives with respect to $\Gamma$ in $L_{0}$ are at constant $Z$ and $Y$, while those with respect to $Z$ and $Y$ in $L_{1}$ are at constant $\Gamma$.

A perturbative solution of the multiscale Liouville Equation (18) is developed such that

$$
\rho=\sum_{n=0}^{\infty} \rho_{n} \varepsilon^{n}
$$

To $O\left(\varepsilon^{0}\right)$, we seek quasi-equilibrium solutions such that $L_{0} \rho_{0}=0$, i.e. $\rho_{0}$ is equilibrated with respect to the atomistic variables $\Gamma$ and hence is independent of $t_{0}$. Introducing a "nanocanonical ensemble" as generalized from that in Ref. [33] for the 
present problem, we obtain

$$
\rho_{0}=\frac{\mathrm{e}^{-\beta H}}{Q(\beta, Z, \Upsilon)} W(Z, \Upsilon, \underline{t}) \equiv \hat{\rho} W,
$$

where $W$ is the reduced probability density and $H$ is the total energy. $H$ is defined as

$$
H=\sum_{i=1}^{N} \frac{p_{i}^{2}}{2 m_{i}}+V\left(\Gamma_{\mathrm{r}}\right)
$$

where $V\left(\Gamma_{\mathrm{r}}\right)$ is the $N$-atom potential energy.

The partition function $Q$ is given by

$$
Q=\int \mathrm{d} \Gamma^{\prime} \Delta\left(\Gamma^{\prime}, \mathrm{Z}, \Upsilon\right) \mathrm{e}^{-\beta H^{\prime}},
$$

where

$$
\Delta\left(\Gamma^{\prime}, Z, \Upsilon\right)=\delta\left(Z-Z^{\prime}\right) \delta\left(\Upsilon-\Upsilon^{\prime}\right),
$$

where $H^{\prime}, Z$ and $Y^{\prime}$ are the $\Gamma^{\prime}$-dependent values of $H, Z$ and $Y$. Further discussion of (22) for a simpler problem is provided elsewhere [37].

To $O(\varepsilon)$, the Liouville equation implies

$$
\left(\frac{\partial}{\partial t_{0}}-L_{0}\right) \rho_{1}=-\frac{\partial \rho_{0}}{\partial t_{1}}+L_{1} \rho_{0} .
$$

As we have assumed that initially $\rho$ is near equilibrium, $\rho_{0}$ contains all the initial (i.e. $t_{0}=0$ ) information; thus, $\rho_{1}$ is zero at $t_{0}=0$. With this, using (20) for $L_{1}$, and recalling that $L_{0} \hat{\rho}=0$, we find

$$
\begin{aligned}
\rho_{1}= & -t_{0} \hat{\rho} \frac{\partial W}{\partial t_{1}}-\int_{0}^{t_{0}} \mathrm{~d} t_{0}^{\prime} \mathrm{e}^{L_{0}\left(t_{0}-t_{0}^{\prime}\right)}\left[\frac{\vec{P}}{m} \cdot \frac{\partial}{\partial \vec{R}}+\vec{f} \cdot \frac{\partial}{\partial \vec{P}}+\frac{\Pi}{m} \frac{\partial}{\partial \Phi}\right. \\
& \left.+g \frac{\partial}{\partial \Pi}+\frac{\vec{P}_{\mathrm{d}}}{m_{\mathrm{d}}} \cdot \frac{\partial}{\partial \vec{R}_{\mathrm{d}}}+\vec{f}_{\mathrm{d}} \cdot \frac{\partial}{\partial \vec{P}_{\mathrm{d}}}+\frac{\Pi_{\mathrm{d}}}{m_{\mathrm{d}}} \frac{\partial}{\partial \Lambda}+h \frac{\partial}{\partial \Pi_{\mathrm{d}}}\right] \hat{\rho} W .
\end{aligned}
$$

The statistical mechanical postulate "the longtime and ensemble averages for equilibrium systems are equal" is assumed and implies

$$
\lim _{t \rightarrow \infty} \frac{1}{t} \int_{-t}^{0} \mathrm{~d} s\left(\mathrm{e}^{-L_{0} s} A\right)=\int \mathrm{d} \Gamma^{\prime} \hat{\rho} \Delta A\left(\Gamma^{\prime}\right) \equiv A^{\text {th }}
$$

for any dynamical variable $A(\Gamma)$.

By multiplying both sides of Equation (27) by $\Delta$, integrating with respect to $\Gamma$, and removing secular behaviour in $\rho_{1}$ (i.e. balancing terms that are divergent at large $t_{0}$ ), we obtain

$$
\begin{aligned}
\rho_{1}=- & \hat{\rho} \int_{0}^{t_{0}} \mathrm{~d} t_{0}^{\prime} \mathrm{e}^{L_{0}\left(t_{0}-t_{0}^{\prime}\right)}\left\{\left(\vec{f}-\vec{f}^{\text {th }}\right) \cdot\left(\beta \frac{\vec{P}}{m}+\frac{\partial}{\partial \vec{P}}\right)+\left(g-g^{\text {th }}\right)\left(\beta \frac{\Pi}{m}+\frac{\partial}{\partial \Pi}\right)\right. \\
& \left.+\left(\vec{f}_{\mathrm{d}}-\vec{f}_{\mathrm{d}}^{\text {th }}\right) \cdot\left(\beta \frac{\vec{P}_{\mathrm{d}}}{m_{\mathrm{d}}}+\frac{\partial}{\partial \vec{P}_{\mathrm{d}}}\right)+\left(h-h^{\text {th }}\right)\left(\beta \frac{\Pi_{\mathrm{d}}}{m_{\mathrm{d}}}+\frac{\partial}{\partial \Pi_{\mathrm{d}}}\right)\right\} W .
\end{aligned}
$$


In the above equation, we used the expressions for $\partial \hat{\rho} / \partial Z$ [Equations (66)-(69)] and $\partial \hat{\rho} / \partial \Upsilon$ [Equations (82)-(85)] derived in Appendix A.

An equation for $W$ can be obtained as follows. Define the reduced probability density $\tilde{W}$ via

$$
\tilde{W}(\mathrm{Z}, \Upsilon, t)=\int \mathrm{d} \Gamma^{\prime} \Delta\left(\Gamma^{\prime}, Z, \Upsilon\right) \rho\left(\Gamma^{\prime}, t\right)
$$

Using the chain rule, properties of the delta function, integration by parts, and the Liouville equation, we obtain

$$
\begin{aligned}
\frac{\partial \tilde{W}}{\partial t}= & -\varepsilon\left\{\frac{\partial}{\partial \vec{R}} \cdot \int \mathrm{d} \Gamma^{\prime} \Delta \rho \frac{\vec{P}^{\prime}}{m}+\frac{\partial}{\partial \vec{P}} \cdot \int \mathrm{d} \Gamma^{\prime} \Delta \rho \vec{f}+\frac{\partial}{\partial \vec{R}_{\mathrm{d}}} \cdot \int \mathrm{d} \Gamma^{\prime} \Delta \rho \frac{\vec{P}_{\mathrm{d}}^{\prime}}{m_{\mathrm{d}}}+\frac{\partial}{\partial \vec{P}_{\mathrm{d}}} \cdot \int \mathrm{d} \Gamma^{\prime} \Delta \rho \overrightarrow{f_{\mathrm{d}}}\right. \\
& \left.+\frac{\partial}{\partial \Phi} \int \mathrm{d} \Gamma^{\prime} \Delta \rho \frac{\Pi^{\prime}}{m}+\frac{\partial}{\partial \Pi} \int \mathrm{d} \Gamma^{\prime} \Delta \rho g+\frac{\partial}{\partial \Lambda} \int \mathrm{d} \Gamma^{\prime} \Delta \rho \frac{\Pi_{\mathrm{d}}^{\prime}}{m_{\mathrm{d}}}+\frac{\partial}{\partial \Pi_{\mathrm{d}}} \int \mathrm{d} \Gamma^{\prime} \Delta \rho h\right\} .
\end{aligned}
$$

Thus, to compute $\partial \tilde{W} / \partial t$ to $O\left(\varepsilon^{2}\right)$ we only require $\rho_{0}$ and $\rho_{1}$. Using Equation (29) for $\rho_{1}$ and Equation (22) for $\rho_{0}$, upon noting that $\tilde{W} \rightarrow W$ as $\varepsilon \rightarrow 0$, we find

$$
\frac{\partial W}{\partial t}=\varepsilon D^{\prime} W,
$$

where

$$
\begin{aligned}
D^{\prime}= & D-\left[\frac{\vec{P}}{m} \cdot \frac{\partial}{\partial \vec{R}}+\vec{f}^{\text {th }} \cdot \frac{\partial}{\partial \vec{P}}+\frac{\Pi}{m} \frac{\partial}{\partial \Phi}+g^{\text {th }} \frac{\partial}{\partial \Pi}\right. \\
& \left.+\frac{\vec{P}_{\mathrm{d}}}{m_{\mathrm{d}}} \cdot \frac{\partial}{\partial \vec{R}_{\mathrm{d}}}+\vec{f}_{\mathrm{d}}^{\text {th }} \cdot \frac{\partial}{\partial \vec{P}_{\mathrm{d}}}+\frac{\Pi_{\mathrm{d}}}{m_{\mathrm{d}}} \frac{\partial}{\partial \Lambda}+h^{\text {th }} \frac{\partial}{\partial \Pi_{\mathrm{d}}}\right]
\end{aligned}
$$

and $D$ is defined as follows

$$
\begin{aligned}
D= & \vec{\gamma}_{f f} \frac{\partial}{\partial \vec{P}} \cdot\left(\beta \frac{\vec{P}}{m}+\frac{\partial}{\partial \vec{P}}\right)+\vec{\gamma}_{f g} \cdot \frac{\partial}{\partial \vec{P}}\left(\beta \frac{\Pi}{m}+\frac{\partial}{\partial \Pi}\right)+\vec{\gamma}_{f f_{\mathrm{d}}} \frac{\partial}{\partial \vec{P}} \cdot\left(\beta \frac{\vec{P}_{\mathrm{d}}}{m_{\mathrm{d}}}+\frac{\partial}{\partial \vec{P}_{\mathrm{d}}}\right) \\
& +\vec{\gamma}_{f h} \cdot \frac{\partial}{\partial \vec{P}}\left(\beta \frac{\Pi_{\mathrm{d}}}{m_{\mathrm{d}}}+\frac{\partial}{\partial \Pi_{\mathrm{d}}}\right)+\vec{\gamma}_{g f} \cdot \frac{\partial}{\partial \Pi}\left(\beta \frac{\vec{P}}{m}+\frac{\partial}{\partial \vec{P}}\right)+\gamma_{g g} \frac{\partial}{\partial \Pi}\left(\beta \frac{\Pi}{m}+\frac{\partial}{\partial \Pi}\right) \\
& +\vec{\gamma}_{g f_{\mathrm{d}}} \cdot \frac{\partial}{\partial \Pi}\left(\beta \frac{\vec{P}_{\mathrm{d}}}{m_{\mathrm{d}}}+\frac{\partial}{\partial \vec{P}_{\mathrm{d}}}\right)+\gamma_{g h} \frac{\partial}{\partial \Pi}\left(\beta \frac{\Pi_{\mathrm{d}}}{m_{\mathrm{d}}}+\frac{\partial}{\partial \Pi_{\mathrm{d}}}\right)+\vec{\gamma}_{f_{\mathrm{d}}} \frac{\partial}{\partial \vec{P}_{\mathrm{d}}} \cdot\left(\beta \frac{\vec{P}}{m}+\frac{\partial}{\partial \vec{P}}\right) \\
& +\vec{\gamma}_{f \mathrm{~d} g} \cdot \frac{\partial}{\partial \vec{P}_{\mathrm{d}}}\left(\beta \frac{\Pi}{m}+\frac{\partial}{\partial \Pi}\right)+{\overrightarrow{\gamma_{f d}}}_{f \mathrm{~d}} \frac{\partial}{\partial \vec{P}_{\mathrm{d}}} \cdot\left(\beta \frac{\vec{P}_{\mathrm{d}}}{m_{\mathrm{d}}}+\frac{\partial}{\partial \vec{P}_{\mathrm{d}}}\right)+\vec{\gamma}_{f_{\mathrm{d}} h} \cdot \frac{\partial}{\partial \vec{P}_{\mathrm{d}}}\left(\beta \frac{\Pi_{\mathrm{d}}}{m_{\mathrm{d}}}+\frac{\partial}{\partial \Pi_{\mathrm{d}}}\right) \\
& +\vec{\gamma}_{h f} \cdot \frac{\partial}{\partial \Pi_{\mathrm{d}}}\left(\beta \frac{\vec{P}}{m}+\frac{\partial}{\partial \vec{P}}\right)+\gamma_{h g} \frac{\partial}{\partial \Pi_{\mathrm{d}}}\left(\beta \frac{\Pi}{m}+\frac{\partial}{\partial \Pi}\right)+\vec{\gamma}_{h f_{\mathrm{d}}} \cdot \frac{\partial}{\partial \Pi_{\mathrm{d}}}\left(\beta \frac{\vec{P}_{\mathrm{d}}}{m_{\mathrm{d}}}+\frac{\partial}{\partial \vec{P}_{\mathrm{d}}}\right) \\
& +\gamma_{h h} \frac{\partial}{\partial \Pi_{\mathrm{d}}}\left(\beta \frac{\Pi_{\mathrm{d}}}{m_{\mathrm{d}}}+\frac{\partial}{\partial \Pi_{\mathrm{d}}}\right) .
\end{aligned}
$$


The $\gamma$-factors are given in Appendix B and mediate frictional exchange of momentum with the host medium and among themselves. This equation provides a theory of the stochastic dynamics of a nanocapsule/payload system. It accounts for the cross-coupling between the various order parameters through the thermal average forces and the friction terms.

The fact that this FP equation is equivalent to a set of eight Langevin equations for $Z$ and $Y$, suggests a way to simulate stochastic payload delivery accounting for fluctuations in the position of the nanocapsule and its structure, as well as that of the payload.

\section{Simulating stochastic payload release}

To illustrate the application of the theory, a series of simulations of drug release from a nanocapsule was carried out. For simplicity, the nanocapsule was assumed to be attached to the target cell surface at the time release was triggered, i.e. the $\mathrm{CM}$ of the nanocapsule was kept constant. The CM of the payload and the nanocapsule structural order parameter were taken to remain constant during release as well. In this case, our model reduces to an FP equation for dispersal only:

$$
\frac{\partial W}{\partial \tau}=-\frac{\Pi_{\mathrm{d}}}{m_{\mathrm{d}}} \frac{\partial W}{\partial \Lambda}+\gamma_{h h} \frac{\partial}{\partial \Pi_{\mathrm{d}}}\left(\beta \frac{\Pi_{\mathrm{d}}}{m_{\mathrm{d}}}+\frac{\partial}{\partial \Pi_{\mathrm{d}}}\right) W,
$$

for $\tau=\varepsilon t$. The equivalent Langevin model allows for practical simulation:

$$
\begin{gathered}
\frac{\mathrm{d} \Lambda}{\mathrm{d} \tau}=\frac{\Pi_{\mathrm{d}}}{m_{\mathrm{d}}}, \\
\frac{\mathrm{d} \Pi_{\mathrm{d}}}{\mathrm{d} \tau}=h^{\text {th }}-\gamma_{h h} \Pi_{\mathrm{d}}+A(\tau),
\end{gathered}
$$

where $A(\tau)$ is a random force whose autocorrelation function is chosen to be consistent with $\gamma$. The objective of this section is to adopt a Monte Carlo approach by repeatedly solving the Langevin equations and then computing the average time course to model a collection of nanocapsules releasing their payloads.

To compare our predictions on release scenario with experimentally observable quantities, a relationship between dispersal and the concentration profile $C(r)$ is introduced:

$$
C(r, \tau)=C_{0}(\tau) \mathrm{e}^{-(r / a \Lambda)^{2}},
$$

where $r$ is the distance from the $\mathrm{CM}$ of the nanocapsule, $a$ is a constant and $C_{0}$ is the payload concentration at the $\mathrm{CM}$ of the nanocapsule. This profile is consistent with the assumption that payload release is spherically symmetric and concentration is a maximum at the $\mathrm{CM}$; it also builds in the meaning of $\Lambda$ which, by definition, is a measure of the radial extent of the cloud of payload molecules. Since there is assumed to be no degradation of payload molecules during dispersal, the total number, $n$, of payload molecules is conserved. This implies

$$
C_{0}=n /(a \sqrt{\pi} \Lambda)^{3},
$$

where $C_{0}$ is assumed to be defined in units of number of molecules per volume. 
The percent of drug release is the quantity most readily comparable to observations; one finds

$$
\% \text { moles released }=\left(\frac{2}{\sqrt{\pi}} y \mathrm{e}^{-y^{2}}+\operatorname{erf}(y)\right) \times 100 \%
$$

as can be computed directly from $C(r)$ by integration over the volume outside the nanocapsule; $y$ is defined below.

The thermal average force $h^{\text {th }}$ appearing in Equation (37) can be found via

$$
h^{\text {th }}=-\partial U / \partial \Lambda,
$$

where $U$ is the potential energy. For the latter, a simple phenomenological expression was adopted

$$
U=4 \pi \int_{0}^{\infty} u C r^{2} \mathrm{~d} r, \quad u=\left\{\begin{array}{l}
\bar{u} R_{\mathrm{c}} \leq r \leq R_{\mathrm{o}} \\
0 \text { otherwise }
\end{array}\right.
$$

where $R_{\mathrm{c}}$ is the inner radius of the nanocapsule and $R_{\mathrm{O}}$ is its outer radius. With this

$$
U=n \bar{u}\left\{\frac{2}{\sqrt{\pi}}\left(x \mathrm{e}^{-x^{2}}-y \mathrm{e}^{-y^{2}}\right)+\operatorname{erf}(x)-\operatorname{erf}(y)\right\},
$$

where $x=R_{\mathrm{c}} / a \Lambda$ and $y=R_{\mathrm{o}} / a \Lambda$

The friction coefficient is a property related to the dispersal via an averaging reflecting the local friction and the overall concentration profile. The following formula was adopted

$$
\begin{gathered}
\gamma=\frac{\int_{0}^{\infty} \tilde{\gamma}(r) C_{0} \mathrm{e}^{-(r / a \Lambda)^{2}} r^{2} \mathrm{~d} r}{\int_{0}^{\infty} C_{0} \mathrm{e}^{-(r / a \Lambda)^{2}} r^{2} \mathrm{~d} r}, \\
\tilde{\gamma}(r)=\left\{\begin{array}{l}
\gamma_{\text {in }} 0 \leq r \leq R_{\mathrm{c}}, \\
\gamma_{\max } R_{\mathrm{c}}<r<R_{\mathrm{o}}, \\
\gamma_{\text {out }} r>R_{\mathrm{o}},
\end{array}\right.
\end{gathered}
$$

where $\gamma_{\max }=g * \mathrm{e}^{\bar{u} / R T}, g *$ is a calibrated coefficient, $\gamma_{\text {in }}, \gamma_{\max }$ and $\gamma_{\text {out }}$ are the maximum friction values in the nanocapsule's cavity, in the outer shell, and in the external medium, respectively.

Drug release in the above model is inhibited by the energy barrier created by the nanocapsule and by friction. Below, we show release profiles computed via the above model for indicated $g *$ values (Figure 2). Values for the radii, masses and number of atoms (see Table 1) are chosen to be consistent with the experimental observations on a typical liposome loaded with doxorubicin [11]. Doxorubicin is an anthracycline antibiotic that is used in chemotherapy and has many acute side effects, particularly a fatal cardiac toxicity. However, cardio toxicity was shown to be reduced for liposome-encapsulated doxorubicin [38] and the rate of release of the drug was found to play an important role in mediating toxicity and improving therapeutic efficacy [39].

As $\gamma_{\max }$ increases with $\bar{u}$, increasing the latter will have similar effect on the release profile as increasing $g *$. Thus, as the barrier height or friction inside the shell increases, the 


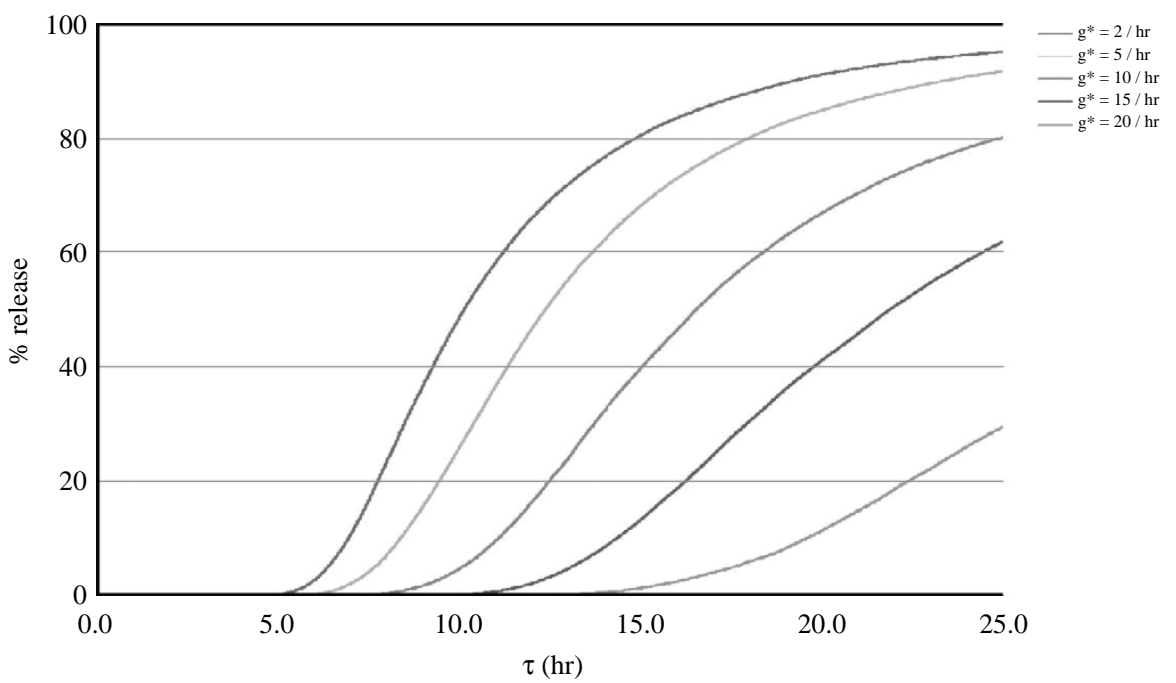

Figure 2. Release profile simulated using Equations (36) and (37) for parameter values as in Table 1.

Table 1. Values used for the simulation.

\begin{tabular}{lcccc}
\hline$n$ (atoms) & $R_{\mathrm{o}}(\mathrm{nm})$ & $R_{\mathrm{c}}(\mathrm{nm})$ & $m *(\mathrm{~g})$ & $m_{d}^{*}(\mathrm{~g})$ \\
\hline $6.7 \times 10^{5}$ & 100 & 92 & $7.208 \times 10^{-17}$ & $9.01 \times 10^{-18}$ \\
\hline
\end{tabular}

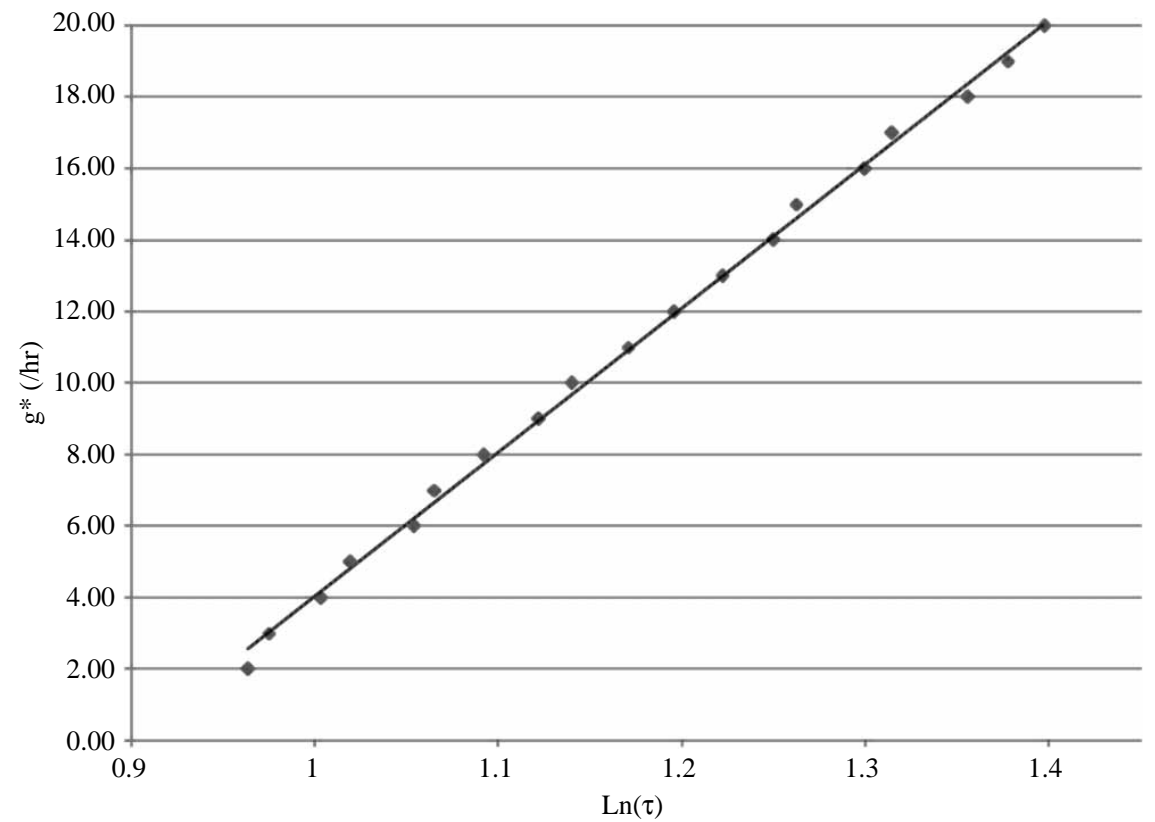

Figure 3. Residence time inside the nanocapsule simulated with different $g^{*}$ values. 
rate of release of drug from the nanocapsule decreases. This is consistent with the fact that increasing the length and/or saturation of the fatty acyl chains comprising a liposome leads to slower release rates. The energy barrier and friction coefficients for the drugnanocapsule interaction can be modified via temperature changes or interaction with the cell membrane. Increasing $\gamma_{\max }$ also leads to longer residence time in the nanocapsule, as shown from the simulation results summarized in Figure 3.

Further discussion of fluctuation effects is provided in Section V.

\section{The stochastic nanoshaker enhanced payload delivery}

Consider the enhancement of the migration and payload delivery induced by the stochastic dynamics of the nanocapsule/payload system. We analyse a special case of the FP equation of Section III that illustrates a nanoshaker enhancement of payload release. Fluctuations are constantly agitating the nanocapsule so that one expects that this could facilitate the escape of payload molecules across the encapsulating membrane. To account for this effect, consider a model cast in terms of the relative position $\vec{R}-\vec{R}_{\mathrm{d}}$ and the dispersal $\Lambda$. We limit the analysis to the case where the nanocapsule/payload system is in an otherwise homogeneous system and no external forces are applied. Define the new order parameters

$$
\begin{gathered}
\vec{R}_{\mathrm{r}}=\vec{R}-\vec{R}_{\mathrm{d}}, \\
\vec{R}_{\mathrm{t}}=\frac{m}{m_{\mathrm{t}}} \vec{R}+\frac{m_{\mathrm{d}}}{m_{\mathrm{t}}} \vec{R}_{\mathrm{d}}, \\
\vec{P}_{\mathrm{r}}=m_{\mathrm{t}}\left[\frac{\vec{P}}{m}-\frac{\vec{P}_{\mathrm{d}}}{m_{\mathrm{d}}}\right], \\
\vec{P}_{\mathrm{t}}=\vec{P}+\vec{P}_{\mathrm{d}},
\end{gathered}
$$

where $m_{\mathrm{t}}=m+m_{\mathrm{d}}$ is the total scaled mass of the nanocapsule/payload composite; $\vec{R}_{\mathrm{r}}$ and $\vec{R}_{\mathrm{t}}$ are the relative and CM positions; and $\vec{P}_{\mathrm{r}}$ and $\vec{P}_{\mathrm{t}}$ are the relative and CM momenta. Defining the forces

$$
\begin{gathered}
\vec{f}_{\mathrm{r}}=\frac{m_{\mathrm{d}}}{m_{\mathrm{t}}} \vec{f}-\frac{m}{m_{\mathrm{t}}} \vec{f}_{\mathrm{d}}, \\
\vec{f}_{\mathrm{t}}=\vec{f}+\vec{f}_{\mathrm{d}},
\end{gathered}
$$

Equation (32) becomes

$$
\begin{aligned}
\frac{\partial W}{\partial t_{1}}= & -\varepsilon\left\{\frac{\vec{P}_{\mathrm{r}}}{m_{\mathrm{t}}} \cdot \frac{\partial}{\partial \vec{R}_{\mathrm{r}}}+K \vec{f}_{\mathrm{r}}^{\text {th }} \cdot \frac{\partial}{\partial \vec{P}_{\mathrm{r}}}+\frac{\vec{P}_{\mathrm{t}}}{m_{\mathrm{t}}} \cdot \frac{\partial}{\partial \vec{R}_{\mathrm{t}}}+\vec{f}_{\mathrm{t}}^{\mathrm{th}} \cdot \frac{\partial}{\partial \vec{P}_{\mathrm{t}}}+\frac{\Pi_{\mathrm{d}}}{m_{\mathrm{d}}} \frac{\partial}{\partial \Lambda}+h^{\text {th }} \frac{\partial}{\partial \Pi_{\mathrm{d}}}\right\} W \\
& +\varepsilon D W
\end{aligned}
$$


and $D$ is rewritten as

$$
\begin{aligned}
D= & K{\overrightarrow{\gamma_{f}}}_{f_{\mathrm{r}}} \cdot \frac{\partial}{\partial \vec{P}_{\mathrm{r}}} \cdot\left(\beta \frac{\vec{P}_{\mathrm{r}}}{m_{\mathrm{t}}}+K \frac{\partial}{\partial \vec{P}_{\mathrm{r}}}\right)+K{\overrightarrow{\gamma_{f}}}_{\mathrm{r}} \cdot \frac{\partial}{\partial \vec{P}_{\mathrm{r}}} \cdot\left(\beta \frac{\vec{P}_{\mathrm{t}}}{m_{\mathrm{t}}}+\frac{\partial}{\partial \vec{P}_{\mathrm{t}}}\right) \\
& +K \vec{\gamma}_{f_{\mathrm{r}}} \cdot \frac{\partial}{\partial \vec{P}_{r}}\left(\beta \frac{\Pi_{\mathrm{d}}}{m_{\mathrm{d}}}+\frac{\partial}{\partial \Pi_{\mathrm{d}}}\right)+\overrightarrow{\vec{\gamma}}_{f_{\mathrm{f}}} \cdot \frac{\partial}{\partial \vec{P}_{\mathrm{t}}} \cdot\left(\beta \frac{\vec{P}_{\mathrm{r}}}{m_{\mathrm{t}}}+K \frac{\partial}{\partial \vec{P}_{\mathrm{r}}}\right) \\
& +\vec{\gamma}_{f_{\mathrm{t}}} \cdot \frac{\partial}{\partial \vec{P}_{\mathrm{t}}} \cdot\left(\beta \frac{\vec{P}_{\mathrm{t}}}{m_{\mathrm{t}}}+\frac{\partial}{\partial \vec{P}_{\mathrm{t}}}\right)+\vec{\gamma}_{f_{\mathrm{t}} h} \cdot \frac{\partial}{\partial \vec{P}_{t}}\left(\beta \frac{\Pi_{\mathrm{d}}}{m_{\mathrm{d}}}+\frac{\partial}{\partial \Pi_{\mathrm{d}}}\right) \\
& +\vec{\gamma}_{h f_{\mathrm{r}}} \cdot \frac{\partial}{\partial \Pi_{\mathrm{d}}}\left(\beta \frac{\vec{P}_{\mathrm{r}}}{m_{\mathrm{t}}}+K \frac{\partial}{\partial \vec{P}_{\mathrm{r}}}\right)+\vec{\gamma}_{h f_{\mathrm{t}}} \cdot \frac{\partial}{\partial \Pi_{\mathrm{d}}}\left(\beta \frac{\vec{P}_{\mathrm{t}}}{m_{\mathrm{t}}}+\frac{\partial}{\partial \vec{P}_{\mathrm{t}}}\right)+\gamma_{h h} \frac{\partial}{\partial \Pi_{\mathrm{d}}}\left(\beta \frac{\Pi_{\mathrm{d}}}{m_{\mathrm{d}}}+\frac{\partial}{\partial \Pi_{\mathrm{d}}}\right)
\end{aligned}
$$

where $K=m_{\mathrm{t}}^{2} / m m_{\mathrm{d}}$ and the friction tensors are given in Appendix B.

Practical simulation of nanocapsule/payload dynamics can be attained via numerical solution of Langevin equations that are equivalent to the FP Equation (52). For the present system, these take the form

$$
\begin{gathered}
\frac{\mathrm{d} R_{\mathrm{r} \alpha}}{\mathrm{d} \tau}=\frac{P_{\mathrm{r} \alpha}}{m_{\mathrm{t}}}, \\
\frac{\mathrm{d} R_{\mathrm{t} \alpha}}{\mathrm{d} \tau}=\frac{P_{\mathrm{t} \alpha}}{m_{\mathrm{t}}}, \\
\frac{\mathrm{d} \Lambda}{\mathrm{d} \tau}=\frac{\Pi_{\mathrm{d}}}{m_{\mathrm{d}}}, \\
\frac{\mathrm{d} P_{\mathrm{r} \alpha}=K\left\{f_{\mathrm{r} \alpha}^{\mathrm{th}}-\frac{\beta}{m_{\mathrm{t}}} \sum_{\hat{\alpha}=1}^{3}\left(\gamma_{f_{\mathrm{r}} \alpha f_{\mathrm{r}} \hat{\alpha}} P_{\mathrm{r} \alpha}+\gamma_{f_{\mathrm{r}} \alpha f_{\mathrm{t}} \hat{\alpha}} P_{t \alpha}\right)-\frac{\beta}{m_{\mathrm{d}}} \gamma_{\mathrm{f}_{\mathrm{r}} \alpha h} \Pi_{\mathrm{d}}+A_{1 \alpha}(\tau)\right\},}{\mathrm{d} P_{\mathrm{t} \alpha}=f_{\mathrm{t} \alpha}^{\mathrm{th}}-\frac{\beta}{m_{\mathrm{t}}} \sum_{\hat{\alpha}=1}^{3}\left(\gamma_{f_{\mathrm{t}} \alpha \mathrm{f}_{\mathrm{r}} \hat{\alpha}} P_{\mathrm{r} \alpha}+\gamma_{f_{\mathrm{t}} \alpha f_{\mathrm{t}} \hat{\alpha}} P_{\mathrm{t} \alpha}\right)-\frac{\beta}{m_{\mathrm{d}}} \gamma_{f_{\mathrm{t}} \alpha h} \Pi_{\mathrm{d}}+A_{2 \alpha}(\tau),} \\
\frac{\mathrm{d} \Pi_{\mathrm{d}}}{\mathrm{d} \tau}=h^{\mathrm{th}}-\frac{\beta}{m_{\mathrm{t}}} \sum_{\hat{\alpha}=1}^{3}\left(\gamma_{h f_{\mathrm{r}} \hat{\alpha}} P_{\mathrm{r} \alpha}+\gamma_{h f_{\mathrm{t}} \hat{\alpha}} P_{t \alpha}\right)-\frac{\beta}{m_{\mathrm{d}}} \gamma_{h h} \Pi_{\mathrm{d}}+A_{3}(\tau),
\end{gathered}
$$

for $\alpha=1,2,3$. The A terms are random forces and are related to the friction coefficients; and

$$
\int_{0}^{\infty} \mathrm{d} \tau \mathrm{d} \tau^{\prime} A_{q \alpha}(\tau) A_{\hat{q} \hat{\alpha}}\left(\tau^{\prime}\right)=2 \gamma_{q \alpha \hat{q} \hat{\alpha}}
$$

for $\alpha, \hat{\alpha}=1,2,3$ and $q, \hat{q}=1,2,3$ referring to $f_{\mathrm{r}}, f_{\mathrm{t}}$ and $h$, respectively.

As can be seen in the above equations and the simulations in Section IV, the fluctuating forces are a necessary element in release phenomena. To avoid premature release, before a nanocapsule reaches the target site its membrane should be able to 
withstand perturbations in the surroundings (i.e. the fluctuating forces are not large enough to overcome the barrier height). To induce the release of payload, fluctuations should be enhanced in order to destabilize the polymer network composing the nanocapsule. This will lead to either decomposing the nanocapsule or increasing the size of the pores to allow the payload to traverse the membrane. Perturbations at the target site can either be intrinsic to the system (i.e. release is triggered by the different environment at the target site) or due to an externally applied field such as heat, light or ultrasound. Nonlinear effects could emerge wherein fluctuations are affected by the molecules of the payload. For example, the local environment created by a payload molecule could widen a channel traversing the membrane. In some cases, such as gene delivery, the payload consists of only a few molecules so that release from a given capsule has a strongly stochastic character. Finally, when average channel width is less than the size of payload molecules, then the rate of escape is determined by the channel expansion/contraction statistics.

\section{Conclusions}

An all-atom, multiscale approach for modelling nanocapsule therapeutic delivery systems has been presented. Order parameters were introduced to characterize special features of these systems, notably the state of the capsule; the dispersal of the therapeutic compound, siRNA, gene, or other payload; and the centre of mass of the payload and nanocapsule. Then, a coarse-grained equation for the stochastic dynamics of these parameters was derived. To illustrate the approach, the time-course of liposomal doxorubicin delivery was simulated. For simplicity, the simulation starts with the fully loaded capsule at the target zone and ready for release. Finally, the nanoshaker effect, i.e. fluctuation-enhanced payload release, was identified.

Benefits of the approach include the following:

- The all-atom description allows for the use of an interatomic force field, thereby avoiding the need for recalibration with each new application.

- Additional order parameters can readily be introduced to account for the presence of a cell surface and other nanoobjects, include other system-specific effects such as externally applied heat, magnetic forces, ligand properties, etc. or provide a more detailed description of the nanocapsule (i.e. shape, orientation or distribution of small-scale structure across the nanocapsule).

In light of the above, we believe that our approach is a starting point for a computer-aided nanomedical design strategy.

Subsequent studies can be done to investigate specific delivery methods using the framework presented here. One can develop modules to estimate the friction coefficients and thermal average forces, thereby enabling a parameter-free modelling approach. Order parameters to describe specific targeted delivery methods and/or release triggers can be added. For example, magnetic forces can be included to account for magnetic nanoparticles used either to guide the capsule to the target zone or as a way to remotely trigger payload release. Also, thermal triggering of the release can be represented by introducing parameters that describe thermal effects and by including reactions in the formalism that allow accounting for thermal breakdown of the capsule and the payload. Finally, a description of the target surface accounting for interaction between the nanocapsule and the cell surface receptors in the target zone can also be included in the formalism. 


\section{Acknowledgements}

We appreciate the support of the U.S. Department of Energy, AFRL, and Indiana University's College of Arts and Sciences and the Office of the Vice President for Research.

\section{References}

[1] S.S. Feng and S. Chien, Chemotherapeutic engineering: Application and further development of chemical engineering principles for chemotherapy of cancer and other diseases, Chem. Eng. Sci. 58(18) (2003), pp. 4087-4114.

[2] J.W. Park, Liposome-based drug delivery in breast cancer treatment, Breast Cancer Res. 4(3) (2002), pp. 93-97.

[3] R. Sinha, G.J. Kim, S.M. Nie, and D.M. Shin, Nanotechnology in cancer therapeutics: Bioconjugated nanoparticles for drug delivery, Mol. Cancer Ther. 5(8) (2006), pp. 1909-1917.

[4] L. Brannon-Peppas and J.O. Blanchette, Nanoparticle and targeted systems for cancer therapy, Adv. Drug Deliv. Rev. 56(11) (2004), pp. 1649-1659.

[5] G.R. Devi, siRNA-based approaches in cancer therapy, Cancer Gene Ther. 13(9) (2006), pp. 819-829.

[6] J. Halder, A.A. Kamat, C.N. Landen, L.Y. Han, S.K. Lutgendorf, Y.G. Lin, W.M. Merritt, N.B. Jennings, A. Chavez-Reyes, R.L. Coleman et al., Focal adhesion kinase targeting using in vivo short interfering RNA delivery in neutral liposomes for ovarian carcinoma therapy, Clin. Cancer Res. 12(16) (2006), pp. 4916-4924.

[7] F. Takeshita and T. Ochiya, Therapeutic potential of RNA interference against cancer, Cancer Sci. 97(8) (2006), pp. 689-696.

[8] T.H. Kim, H. Jin, H.W. Kim, M.H. Cho, and C.S. Cho, Mannosylated chitosan nanoparticlebased cytokine gene therapy suppressed cancer growth in BALB/c mice bearing CT-26 carcinoma cells, Mol. Cancer Ther. 5(7) (2006), pp. 1723-1732.

[9] L.W. Seymour, The future of gene therapy in the UK, Trends Biotechnol. 24(8) (2006), pp. $347-349$

[10] J. Suh, D. Wirtz, and J. Hanes, Efficient active transport of gene nanocarriers to the cell nucleus, Proc. Natl. Acad. Sci. USA 100(7) (2003), pp. 3878-3882.

[11] D.C. Drummond, O. Meyer, K.L. Hong, D.B. Kirpotin, and D. Papahadjopoulos, Optimizing liposomes for delivery of chemotherapeutic agents to solid tumors, Pharmacol. Rev. 51(4) (1999), pp. 691-743.

[12] A. Nagayasu, K. Uchiyama, and H. Kiwada, The size of liposomes: A factor which affects their targeting efficiency to tumors and therapeutic activity of liposomal antitumor drugs, Adv. Drug Deliv. Rev. 40(1-2) (1999), pp. 75-87.

[13] G.J.R. Charrois and T.M. Allen, Drug release rate influences the pharmacokinetics, biodistribution, therapeutic activity, and toxicity of pegylated liposomal doxorubicin formulations in murine breast cancer, Biochim. Biophys. Acta Biomembr. 1663(1-2) (2004), pp. 167-177.

[14] P. Srinath and P.V. Diwan, Stealth liposomes - an overview, Indian J. Pharm. 26 (1994), pp. $179-184$.

[15] B. Uziely, S. Jeffers, R. Isacson, K. Kutsch, D. Weitsao, Z. Yehoshua, E. Libson, F.M. Muggia, and A. Gabizon, Liposomal doxorubicin - activity and unique toxicities during 2 complementary phase-I studies, J. Clin. Oncol. 13(7) (1995), pp. 1777-1785.

[16] D.R. Siwak, A.M. Tari, and G. Lopez-Berestein, The potential of drug-carrying immunoliposomes as anticancer agents, Clin. Cancer Res. 8(4) (2002), pp. 955-956.

[17] J. Huwyler, D.F. Wu, and W.M. Pardridge, Brain drug delivery of small molecules using immunoliposomes, Proc. Natl. Acad. Sci. USA 93(24) (1996), pp. 14164-14169.

[18] E. Forssen and M. Willis, Ligand-targeted liposomes, Adv. Drug Deliv. Rev. 29(3) (1998), pp. 249-271.

[19] A. Khan, Y. Shukla, N. Kalra, M. Alam, M.G. Ahmad, S.R. Hakim, and M. Owais, Potential of diallyl sulfide bearing $\mathrm{pH}$-sensitive liposomes in chemoprevention against DMBA-induced skin papilloma, Mol. Med. 13(7-8) (2007), pp. 443-451.

[20] A.A. Kale and V.P. Torchillin, Enhanced transfection of tumor cells in vivo using 'Smart' pH-sensitive TAT-modified pegylated liposomes, J. Drug Target. 15(7-8) (2007), pp. 538-545.

[21] D.C. Drummond, M. Zignani, and J.C. Leroux, Current status of $\mathrm{pH}$-sensitive liposomes in drug delivery, Prog. Lipid Res. 39(5) (2000), pp. 409-460. 
[22] J. Wells, A. Sen, and S.W. Hui, Localized delivery to CT-26 tumors in mice using thermosensitive liposomes, Int. J. Pharm. 261(1-2) (2003), pp. 105-114.

[23] S. Dromi, V. Frenkel, A. Luk, B. Traughber, M. Angstadt, M. Bur, J. Poff, J.W. Xie, S.K. Libutti, K.C.P. Li et al., Pulsed-high intensity focused ultrasound and low temperature sensitive liposomes for enhanced targeted drug delivery and antitumor effect, Clin. Cancer Res. 13(9) (2007), pp. $2722-2727$.

[24] P. Shum, J.M. Kim, and D.H. Thompson, Phototriggering of liposomal drug delivery systems, Adv. Drug Deliv. Rev. 53(3) (2001), pp. 273-284.

[25] P. Meers, Enzyme-activated targeting of liposomes, Adv. Drug Deliv. Rev. 53(3) (2001), p. 04490.

[26] H. Harashima and H. Kiwada, The pharmacokinetics of liposomes in tumor targeting, Adv. Drug Deliv. Rev. 40 (1999), pp. 39-61.

[27] D.G. Kanjickal and S.T. Lopina, Modeling of drug release from polymeric delivery systems - a review, Crit. Rev. Ther. Drug Carrier Syst. 21(5) (2004), pp. 345-386.

[28] D.O. Cooney, Effect of geometry dissolution of pharmaceutical tablets and other solids surface detachment kinetics controlling, AIChE J. 18(2) (1972), pp. 446-449.

[29] N.A. Peppas, Analysis of Fickian and non-Fickian drug release from polymers, Pharm. Acta Helvetiae 60(4) (1985), pp. 110-111.

[30] P.I. Lee, Diffusional release of a solute from a polymeric matrix - approximate analytical solutions, J. Membr. Sci. 7(3) (1980), pp. 255-275.

[31] A. Göpferich, Bioerodible implants with programmable drug release, J. Control. Release 44(2-3) (1997), pp. 271-281.

[32] P. Ortoleva, Nanoparticle dynamics: A multiscale analysis of the Liouville equation, J. Phys. Chem. B 109(45) (2005), pp. 21258-21266.

[33] Y. Miao and P. Ortoleva, All-atom multiscaling and new ensembles for dynamical nanoparticles, J. Chem. Phys. 125(4) (2006), p. 044901.

[34] - Viral structural transitions: An all-atom multiscale theory, J. Chem. Phys. 125(21) (2006), p. 214901.

[35] Z. Shreif and P. Ortoleva, Curvilinear all-atom multiscale (CAM) theory of macromolecular dynamics, J. Stat. Phys. 130(4) (2008), pp. 669-685.

[36] J. Yang, H. Lee, W. Hyung, S.B. Park, and S. Haam, Magnetic PECA nanoparticles as drug carriers for targeted delivery: Synthesis and release characteristics, J. Microencapsul. 23(2) (2006), pp. 203-212.

[37] S. Pankavich, Z. Shreif, and P. Ortoleva, Multiscaling for classical nanosystems: Derivation of Smoluchowski \& Fokker-Planck equations, Physica A. 387(16-17) (2008), pp. 4053-4069.

[38] G. Batist, G. Ramakrishnan, C.S. Rao, A. Chandrasekharan, J. Gutheil, T. Guthrie, P. Shah, A. Khojasteh, M.K. Nair, K. Hoelzer et al., Reduced cardiotoxicity and preserved antitumor efficacy of liposome-encapsulated doxorubicin and cyclophosphamide compared with conventional doxorubicin and cyclophosphamide in a randomized, multicentre trial of metastatic breast cancer, J. Clin. Oncol. 19(5) (2001), pp. 1444-1454.

[39] T.M. Allen, D.R. Mumbengegwi, and G.J.R. Charrois, Anti-CD19-targeted liposomal doxorubicin improves the therapeutic efficacy in murine B-cell lymphoma and ameliorates the toxicity of liposomes with varying drug release rates, Clin. Cancer Res. 11(9) (2005), pp. $3567-3573$.

\section{Appendix A}

To evaluate the partition function $Q$ arising in the lowest order solution in the multiscale perturbation method of Section III several steps must be taken. First, we express the $\mathrm{N}$-atom potential and kinetic energies in terms of the order parameters and residual effects of the detailed all-atom configuration. We then introduce a Fourier transform method to evaluate the momentum integrations.

The set of atomic positions can be written in terms of coherent and incoherent parts as follows:

$$
\vec{r}_{i}=\Theta_{i}\left(\vec{R}+\vec{\sigma}_{i}+\Phi \overrightarrow{\bar{X}} \hat{s}_{i}^{0}\right)+\Theta_{i}^{\mathrm{d}}\left(\vec{R}_{\mathrm{d}}+\Lambda \hat{s}_{i}^{\mathrm{d}}\right), \quad \Theta_{i}+\Theta_{i}^{\mathrm{d}} \neq 0 .
$$

Introduction of the residual (incoherent) displacement $\vec{\sigma}_{i}$ accounts for fluctuations of the nanocapsule's atoms over-and-above the coherent motion generated by $\Phi$ and $\vec{X}$. For the payload's atoms, there is no relevant reference configuration as during delivery these atoms migrate via a 
random walk relative to each other. The coherent aspect of payload delivery is embedded in $\Lambda$ as it captures the overall range of the random walk.

With this, the derivatives of the $\mathrm{N}$-atom potential energy with respect to the order parameters can be found by using the chain rule and the fact that $(\partial V) /\left(\partial \vec{r}_{i}\right)=-\vec{F}_{i}$

$$
\begin{gathered}
\frac{\partial V}{\partial \vec{R}}=\sum_{i=1}^{N} \frac{\partial V}{\partial \bar{r}_{i}} \Theta_{i}=-\vec{f}, \\
\frac{\partial V}{\partial \vec{R}_{\mathrm{d}}}=\sum_{i=1}^{N} \frac{\partial V}{\partial \bar{r}_{i}} \Theta_{i}^{\mathrm{d}}=-\vec{f}_{\mathrm{d}}, \\
\frac{\partial V}{\partial \Phi}=\sum_{i=1}^{N} \frac{\partial V}{\partial \bar{r}_{i}} \cdot \overrightarrow{\bar{X}} \hat{s}_{i}^{0} \Theta_{i}=-g, \\
\frac{\partial V}{\partial \Lambda}=\sum_{i=1}^{N} \frac{\partial V}{\partial \bar{r}_{i}} \cdot \hat{s}_{i}^{\mathrm{d}} \Theta_{i}^{\mathrm{d}}=-h .
\end{gathered}
$$

Now, proceeding as in Ref. [37], we get

$$
\begin{aligned}
& \frac{\partial \hat{\rho}}{\partial \vec{R}}=-\beta \vec{\rho}^{\text {th }}, \\
& \frac{\partial \hat{\rho}}{\partial \vec{R}_{\mathrm{d}}}=-\beta \vec{\rho}_{\mathrm{d}}^{\mathrm{th}}, \\
& \frac{\partial \hat{\rho}}{\partial \Phi}=-\beta \hat{\rho}^{\mathrm{th}}, \\
& \frac{\partial \hat{\rho}}{\partial \Lambda}=-\beta \hat{\rho} h^{\mathrm{th}} .
\end{aligned}
$$

Now, we can rewrite the partition function in Equation (24) as

$$
Q=Q_{0} Q_{1} Q_{2}
$$

where

$$
\begin{gathered}
Q_{0}=\int \mathrm{d} \Gamma_{\mathrm{p}_{0}}^{\prime} \exp \left\{-\beta \sum_{j=1}^{N} \frac{p_{j}^{2}}{2 m_{j}}\left(1-\Theta_{j}\right)\left(1-\Theta_{j}^{\mathrm{d}}\right)\right\}, \\
Q_{1}=\int \mathrm{d} \Gamma_{\mathrm{r}}^{\prime} \mathrm{d} \Gamma_{\mathrm{p}_{\mathrm{d}}}^{\prime} \mathrm{e}^{-\beta V} \delta\left(\vec{P}_{\mathrm{d}}-\vec{P}_{\mathrm{d}}^{\prime}\right) \delta\left(\Pi_{\mathrm{d}}-\Pi_{\mathrm{d}}^{\prime}\right) \exp \left\{-\beta \sum_{j=1}^{N} \frac{p_{j}^{2}}{2 m_{j}} \Theta_{j}^{\mathrm{d}}\right\}, \\
Q_{2}=\int \mathrm{d} \Gamma_{\mathrm{p}_{\mathrm{c}}}^{\prime} \delta\left(\vec{P}-\vec{P}^{\prime}\right) \delta\left(\Pi-\Pi^{\prime}\right) \exp \left\{-\beta \sum_{j=1}^{N} \frac{p_{j}^{2}}{2 m_{j}} \Theta_{j}\right\},
\end{gathered}
$$

where $\Gamma_{\mathrm{p}_{0}}^{\prime}, \Gamma_{\mathrm{p}_{\mathrm{d}}}^{\prime}$ and $\Gamma_{\mathrm{p}_{\mathrm{c}}}^{\prime}$ are $\Gamma_{\mathrm{p}}^{\prime}$ for the medium, payload and nanoparticle atoms. 
Using the Fourier transform we get

$$
\begin{aligned}
\Delta\left(\vec{P}-\vec{P}^{\prime}\right) \delta\left(\Pi-\Pi^{\prime}\right)= & \frac{1}{(2 \pi)^{2}} \int_{-\infty}^{\infty} \mathrm{d} k_{1} \exp \left\{\mathrm{i} k_{1}\left[P_{x}-\varepsilon \sum_{j=1}^{N} p_{j x} \Theta_{j}\right]\right\} \\
& \int_{-\infty}^{\infty} \mathrm{d} k_{2} \exp \left\{\mathrm{i} k_{2}\left[P_{y}-\varepsilon \sum_{j=1}^{N} p_{j y} \Theta_{j}\right]\right\} \int_{-\infty}^{\infty} \mathrm{d} k_{3} \exp \left\{\mathrm{i} k_{3}\left[P_{z}-\varepsilon \sum_{j=1}^{N} p_{j z} \Theta_{j}\right]\right\} \\
& \int_{-\infty}^{\infty} \mathrm{d} k_{4} \exp \left\{\mathrm { i } k _ { 4 } \left[\Pi-\varepsilon \overrightarrow{\bar{X}} \sum_{j=1}^{N}\left(p_{j x} \hat{s}_{j x}^{0}+p_{j y} \hat{s}_{j y}^{0}+p_{j z} \hat{s}_{j z}^{0}\right) \Theta_{j}\right.\right. \\
& \left.\left.+\varepsilon^{3} \overrightarrow{\bar{X}} \sum_{j=1}^{N}\left(p_{j x} \sum_{\ell=1}^{N} m_{\ell} \hat{s}_{\ell x}^{0}+p_{j y} \sum_{\ell=1}^{N} m_{\ell} \hat{s}_{\ell y}^{0}+p_{j z} \sum_{\ell=1}^{N} m_{\ell} \hat{s}_{\ell z}^{0}\right) \Theta_{j}\right]\right\}
\end{aligned}
$$

Inserting Equation (74) in Equation (73) we get

$$
\begin{aligned}
Q_{2}= & \frac{1}{(2 \pi)^{2}} \int \mathrm{d} k_{1} \mathrm{~d} k_{2} \mathrm{~d} k_{3} \mathrm{~d} k_{4}\left\{\exp \left[\mathrm{i} k_{1} P_{x}\right] \exp \left[\mathrm{i} k_{2} P_{y}\right] \exp \left[\mathrm{i} k_{3} P_{z}\right] \exp \left[\mathrm{i} k_{4} \Pi\right]\right. \\
& \int \mathrm{d} p_{1 x} \exp \left(-\beta \frac{p_{1 x}^{2}}{2 m_{1}}\right) \exp \left[-\mathrm{i}\left(\varepsilon k_{1}+\varepsilon \overrightarrow{\vec{X}} k_{4} \hat{s}_{1 x}^{0}-\varepsilon \overrightarrow{\vec{X}} \sum_{\ell=1}^{N_{\mathrm{c}}} m_{\ell} \hat{s}_{\ell x}^{0}\right) p_{1 x}\right] \\
& \int \mathrm{d} p_{2 x} \exp \left(-\beta \frac{p_{2 x}^{2}}{2 m_{2}}\right) \exp \left[-\mathrm{i}\left(\varepsilon k_{1}+\varepsilon \overrightarrow{\bar{X}} k_{4} \hat{S}_{2 x}^{0}-\varepsilon^{3} \overrightarrow{\vec{X}} \sum_{\ell=1}^{N_{\mathrm{c}}} m_{\ell} \hat{s}_{\ell x}^{0}\right) p_{2 x}\right] \\
& \ldots \int \mathrm{d} p_{1 y} \exp \left(-\beta \frac{p_{1 y}^{2}}{2 m_{1}}\right) \exp \left[-\mathrm{i}\left(\varepsilon k_{2}+\varepsilon \overrightarrow{\bar{X}} k_{4} \hat{S}_{1 y}^{0}-\varepsilon^{3} \overrightarrow{\bar{X}} \sum_{\ell=1}^{N_{\mathrm{c}}} m_{\ell} \hat{s}_{\ell y}^{0}\right) p_{1 y}\right] \\
& \ldots \int \mathrm{d} p_{1 z} \exp \left(-\beta \frac{p_{1 z}^{2}}{2 m_{1}}\right) \exp \left[-\mathrm{i}\left(\varepsilon k_{3}+\varepsilon \overrightarrow{\bar{X}} k_{4} \hat{s}_{1 z}^{0}-\varepsilon^{3} \overrightarrow{\bar{X}} \sum_{\ell=1}^{N_{\mathrm{c}}} m_{\ell} \hat{s}_{\ell z}^{0}\right) p_{1 z}\right] \\
& \left.\ldots \int \mathrm{d} p_{N_{\mathrm{c}} z} \exp \left(-\beta \frac{p_{N_{\mathrm{c}} z}^{2}}{2 m_{N_{\mathrm{c}}}}\right) \exp \left[-\mathrm{i}\left(\varepsilon k_{3}+\varepsilon \overrightarrow{\bar{X}} k_{4} \hat{s}_{N_{\mathrm{c}} z}^{0}-\varepsilon^{3} \overrightarrow{\vec{X}} \sum_{\ell=1}^{N_{\mathrm{c}}} m_{\ell} \hat{s}_{\ell z}^{0}\right) p_{N_{c} z}\right]\right\}
\end{aligned}
$$

where $N_{\mathrm{c}}$ is the number of atoms on the nanocapsule.

For simplicity, we take the reference nanocapsule configuration to be symmetric (i.e. $\sum_{i=1}^{N_{\mathrm{c}}} m_{i} \hat{s}_{i \alpha}^{0}=0$ for $\alpha=x, y, z$ ). With this, and using the inverse Fourier transform

$$
\exp \left(-\lambda k^{2}\right)=\frac{1}{\sqrt{2 \pi}} \int_{-\infty}^{\infty} \frac{1}{\sqrt{4 \pi \lambda}} \exp (-\mathrm{i} k z) \exp \left(\frac{-z^{2}}{4 \lambda}\right) \mathrm{d} z
$$


$Q_{2}$ takes the form

$$
\begin{aligned}
Q_{2}= & \frac{C^{3}}{(2 \pi)^{2}} \int_{-\infty}^{\infty} \mathrm{d} k_{1} \exp \left(\mathrm{i} k_{1} P_{x}\right) \exp \left(-\frac{m}{2 \beta} k_{1}^{2}\right) \int_{-\infty}^{\infty} \mathrm{d} k_{2} \exp \left(\mathrm{i} k_{2} P_{y}\right) \exp \left(-\frac{m}{2 \beta} k_{2}^{2}\right) \\
& \int_{-\infty}^{\infty} \mathrm{d} k_{3} \exp \left(\mathrm{i} k_{3} P_{z}\right) \exp \left(-\frac{m}{2 \beta} k_{3}^{2}\right) \int_{-\infty}^{\infty} \mathrm{d} k_{4} \exp \left(\mathrm{i} k_{4} \Pi\right) \exp \left(-\frac{m}{2 \beta} \overline{\bar{X}}^{2} k_{4}^{2}\right) \\
& =C^{\prime} \exp \left(-\beta \frac{P^{2}}{2 m}\right) \exp \left(-\beta \frac{\Pi^{2}}{2 m \overline{\bar{X}}^{2}}\right),
\end{aligned}
$$

where $C=2 \pi \sqrt{\frac{m_{1}}{\beta}} \cdots 2 \pi \sqrt{\frac{m_{N_{\mathrm{c}}}}{\beta}} ; C^{\prime}=\frac{C}{\overline{\bar{X}}}\left(\frac{2 \pi \beta}{m}\right)^{2}$.

Similarly

$$
\begin{aligned}
\delta\left(\vec{P}_{\mathrm{d}}-\vec{P}_{\mathrm{d}}^{\prime}\right) \delta\left(\Pi_{\mathrm{d}}-\Pi_{\mathrm{d}}^{\prime}\right)= & \frac{1}{(2 \pi)^{2}} \int_{-\infty}^{\infty} \mathrm{d} k_{1} \exp \left\{\mathrm{i} k_{1}\left[P_{\mathrm{d} x}-\varepsilon \sum_{j=1}^{N} p_{j x} \Theta_{j}^{\mathrm{d}}\right]\right\} \\
& \int_{-\infty}^{\infty} \mathrm{d} k_{2} \exp \left\{\mathrm{i} k_{2}\left[P_{\mathrm{d} y}-\varepsilon \sum_{j=1}^{N} p_{j y} \Theta_{j}^{\mathrm{d}}\right]\right\} \\
& \int_{-\infty}^{\infty} \mathrm{d} k_{3} \exp \left\{\mathrm{i} k_{3}\left[P_{\mathrm{d} z}-\varepsilon \sum_{j=1}^{N} p_{j z} \Theta_{j}^{\mathrm{d}}\right]\right\} \\
& \int_{-\infty}^{\infty} \mathrm{d} k_{4} \exp \left\{\mathrm { i } k _ { 4 } \left[\Pi_{\mathrm{d}}-\varepsilon \sum_{j=1}^{N}\left(p_{j x} \hat{S}_{j x}^{\mathrm{d}}+p_{j y} \hat{s}_{j y}^{\mathrm{d}}+p_{j z} \hat{S}_{j z}^{\mathrm{d}}\right) \Theta_{j}^{\mathrm{d}}\right.\right. \\
+ & \left.\left.\varepsilon^{3} \sum_{j=1}^{N}\left(p_{j x} \sum_{\ell=1}^{N} m_{\ell} \hat{s}_{\ell x}^{\mathrm{d}}+p_{j y} \sum_{\ell=1}^{N} m_{\ell} \hat{s}_{t y}^{\mathrm{d}}+p_{j z} \sum_{\ell=1}^{N} m_{\ell} \hat{s}_{\ell z}^{\mathrm{d}}\right) \Theta_{j}\right]\right\} .
\end{aligned}
$$

Thus,

$$
\begin{aligned}
Q_{1}= & \frac{1}{(2 \pi)^{2}} \int \mathrm{d} \Gamma_{\mathrm{r}}^{\prime} \mathrm{e}^{-\beta V} \mathrm{~d} k_{1} \mathrm{~d} k_{2} \mathrm{~d} k_{3} \mathrm{~d} k_{4}\left\{\exp \left[\mathrm{i} k_{1} P_{\mathrm{d} x}\right] \exp \left[\mathrm{i} k_{2} P_{\mathrm{dy}}\right] \exp \left[\mathrm{i} k_{3} P_{\mathrm{d} z}\right]\right. \\
& \exp \left[\mathrm{i} k_{4} \Pi_{\mathrm{d}}\right] \int \mathrm{d} p_{1 x} \exp \left(-\beta \frac{p_{1 x}^{2}}{2 m_{1}}\right) \exp \left[-\mathrm{i}\left(\varepsilon k_{1}+\varepsilon k_{4} \hat{S}_{1 x}^{\mathrm{d}}-\varepsilon^{3} \sum_{\ell=1}^{N_{\mathrm{d}}} m_{\ell} \hat{S}_{\ell x}^{\mathrm{d}}\right) p_{1 x}\right] \\
& \int \mathrm{d} p_{2 x} \exp \left(-\beta \frac{p_{2 x}^{2}}{2 m_{2}}\right) \exp \left[-\mathrm{i}\left(\varepsilon k_{1}+\varepsilon k_{4} \hat{s}_{2 x}^{\mathrm{d}}-\varepsilon^{3} \sum_{\ell=1}^{N_{\mathrm{d}}} m_{\ell} \hat{s}_{\ell x}^{0}\right) p_{2 x}\right] \\
& \ldots \int \mathrm{d} p_{1 y} \exp \left(-\beta \frac{p_{1 y}^{2}}{2 m_{1}}\right) \exp \left[-\mathrm{i}\left(\varepsilon k_{2}+\varepsilon k_{4} \hat{s}_{1 y}^{\mathrm{d}}-\varepsilon^{3} \sum_{\ell=1}^{N_{\mathrm{d}}} m_{\ell} \hat{s}_{\ell y}^{\mathrm{d}}\right) p_{1 y}\right] \\
& \ldots \int \mathrm{d} p_{1 z} \exp \left(-\beta \frac{p_{1 z}^{2}}{2 m_{1}}\right) \exp \left[-\mathrm{i}\left(\varepsilon k_{3}+\varepsilon k_{4} \hat{s}_{1 z}^{\mathrm{d}}-\varepsilon^{3} \sum_{\ell=1}^{N_{\mathrm{d}}} m_{\ell} \hat{\hat{S}}_{\ell z}^{\mathrm{d}}\right) p_{1 z}\right] \\
& \left.\ldots \int \mathrm{d} p_{N_{\mathrm{d} z}} \exp \left(-\beta \frac{p_{N_{\mathrm{d}} z}^{2}}{2 m_{N_{\mathrm{c}}}}\right) \exp \left[-\mathrm{i}\left(\varepsilon k_{3}+\varepsilon k_{4} \mathrm{~s}_{N_{\mathrm{d}} z}^{\mathrm{d}}-\varepsilon^{3} \sum_{\ell=1}^{N_{\mathrm{d}}} m_{\ell} \hat{s}_{\ell z}^{\mathrm{d}}\right) p_{N_{\mathrm{d} z} z}\right]\right\}
\end{aligned}
$$

where $N_{\mathrm{d}}$ is the number of atoms on the payload. In addition, the indices are renumbered in the above equation. 
After release, payload atoms would go in all directions and therefore $\sum_{\ell=1}^{N_{\mathrm{d}}} m_{\ell} \hat{s}_{\ell \alpha}^{\mathrm{d}}$ is approximately zero. Following the same method as above, we get

$$
Q_{1}=C^{\prime \prime} \int \mathrm{d} \Gamma_{\mathrm{r}}^{\prime} \mathrm{e}^{-\beta V} \exp \left(-\beta \frac{P_{\mathrm{d}}^{2}}{2 m}\right) \exp \left(-\beta \frac{\Pi_{\mathrm{d}}^{2}}{2 m}\right)
$$

where $C^{\prime \prime}=\left(2 \pi \sqrt{\frac{m_{1}}{\beta}} \cdots 2 \pi \sqrt{\frac{m_{N_{\mathrm{d}}}}{\beta}}\right)\left(\frac{2 \pi \beta}{m_{\mathrm{d}}}\right)$.

Noting that $P_{\mathrm{d}}$ and $\Pi_{\mathrm{d}}$ are independent of $\Gamma^{\prime}$ and ignoring $\overrightarrow{\vec{X}}$ (which is consistent with Section III), we get

$$
Q=C^{\prime} C^{\prime \prime} Q_{0}\left(\int \mathrm{d} \Gamma_{\mathrm{r}}^{\prime} \mathrm{e}^{-\beta V}\right) \exp \left(-\beta \frac{P^{2}}{2 m}\right) \exp \left(-\beta \frac{\Pi^{2}}{2 m}\right) \exp \left(-\beta \frac{P_{\mathrm{d}}^{2}}{2 m}\right) \exp \left(-\beta \frac{\Pi_{\mathrm{d}}^{2}}{2 m}\right) .
$$

With this, we get

$$
\begin{gathered}
\frac{\partial \hat{\rho}}{\partial \vec{P}}=\beta \hat{\rho} \frac{\vec{P}}{m}, \\
\frac{\partial \hat{\rho}}{\partial \Pi}=\beta \hat{\rho} \frac{\Pi}{m}, \\
\frac{\partial \hat{\rho}}{\partial \vec{P}_{\mathrm{d}}}=\beta \hat{\rho} \frac{\vec{P}_{\mathrm{d}}}{m_{\mathrm{d}}}, \\
\frac{\partial \hat{\rho}}{\partial \Pi_{\mathrm{d}}}=\beta \hat{\rho} \frac{\Pi_{\mathrm{d}}}{m_{\mathrm{d}}},
\end{gathered}
$$

\section{Appendix B}

Multiple scale analyses have focused on two extreme cases - inertial and friction dominated dynamics. In the latter, one obtains Smoluchowsky equations which do not track the momentum variables. In the present formulation, we attempted to balance both tendencies. This can be accomplished when either the inertial terms are small (i.e. entering in $O(\varepsilon)$ in the perturbative analysis of the Liouville equation, or the friction coefficients are large (i.e $O\left(\varepsilon^{-1}\right)$ ). In the following, the second approach is considered.

Assume the phenomena under study to be characterized by long-lived modes such that the integrated generalized-force correlation functions are $O\left(\varepsilon^{-1}\right)$ and not $O\left(\varepsilon^{0}\right)$. We thus introduce $\gamma$ - 
factors as follows:

$$
\begin{aligned}
& \gamma_{f f, k l} \equiv \varepsilon \int_{-\infty}^{0} \mathrm{~d} t\left\{\left(f_{k}(0) f_{l}(t)\right)^{\mathrm{th}}-f_{k}^{\mathrm{th}} f_{l}^{\mathrm{th}}\right\} \\
& \gamma_{f g, k} \equiv \varepsilon \int_{-\infty}^{0} \mathrm{~d} t\left\{\left(f_{k}(0) g(t)\right)^{\mathrm{th}}-f_{k}^{\mathrm{th}} g^{\mathrm{th}}\right\}, \\
& \gamma_{f f f_{\mathrm{d},}, l} \equiv \varepsilon \int_{-\infty}^{0} \mathrm{~d} t\left\{\left(f_{k}(0) f_{\mathrm{d} l}(t)\right)^{\mathrm{th}}-f_{k}^{\mathrm{th}} f_{\mathrm{d} l}^{\mathrm{th}}\right\}, \\
& \gamma_{f h, k} \equiv \varepsilon \int_{-\infty}^{0} \mathrm{~d} t\left\{\left(f_{k}(0) h(t)\right)^{\mathrm{th}}-f_{k}^{\mathrm{th}} h^{\text {th }}\right\}, \\
& \gamma_{g f, k} \equiv \varepsilon \int_{-\infty}^{0} \mathrm{~d} t\left\{\left(g(0) f_{k}(t)\right)^{\mathrm{th}}-g^{\mathrm{th}} f_{k}^{\mathrm{th}}\right\}, \\
& \gamma_{g g} \equiv \varepsilon \int_{-\infty}^{0} \mathrm{~d} t\left\{(g(0) g(t))^{\mathrm{th}}-g^{\mathrm{th}} g^{\mathrm{th}}\right\} \\
& \gamma_{g f_{\mathrm{d}}, k} \equiv \varepsilon \int_{-\infty}^{0} \mathrm{~d} t\left\{\left(g(0) f_{\mathrm{d} k}(t)\right)^{\mathrm{th}}-g^{\mathrm{th}} f_{\mathrm{d} k}^{\mathrm{th}}\right\}, \\
& \gamma_{g h} \equiv \varepsilon \int_{-\infty}^{0} \mathrm{~d} t\left\{(\mathrm{~g}(0) h(t))^{\mathrm{th}}-g^{\mathrm{th}} h^{\mathrm{th}}\right\} \\
& \gamma_{f_{\mathrm{d}} f, k l} \equiv \varepsilon \int_{-\infty}^{0} \mathrm{~d} t\left\{\left(f_{\mathrm{d} k}(0) f_{l}(t)\right)^{\mathrm{th}}-f_{\mathrm{d} k}^{\mathrm{th}} f_{l}^{\mathrm{th}}\right\}, \\
& \gamma_{f_{\mathrm{d} g} g, k} \equiv \varepsilon \int_{-\infty}^{0} \mathrm{~d} t\left\{\left(f_{\mathrm{d} k}(0) g(t)\right)^{\mathrm{th}}-f_{\mathrm{d} k}^{\mathrm{th}} g^{\mathrm{th}}\right\}, \\
& \gamma_{f_{\mathrm{d}} f_{\mathrm{d}}, k l} \equiv \varepsilon \int_{-\infty}^{0} \mathrm{~d} t\left\{\left(f_{\mathrm{d} k}(0) f_{\mathrm{d} l}(t)\right)^{\text {th }}-f_{\mathrm{d} k}^{\mathrm{th}} f_{\mathrm{d} l}^{\mathrm{th}}\right\}, \\
& \gamma_{f_{\mathrm{d}} h, k} \equiv \varepsilon \int_{-\infty}^{0} \mathrm{~d} t\left\{\left(f_{\mathrm{d} k}(0) h(t)\right)^{\mathrm{th}}-f_{\mathrm{d} k}^{\mathrm{th}} h^{\mathrm{th}}\right\}, \\
& \gamma_{f_{\mathrm{r}} f_{\mathrm{r}}, k l} \equiv \varepsilon \int_{-\infty}^{0} d t\left\{\left(f_{\mathrm{r} k}(0) f_{\mathrm{r} l}(t)\right)^{\mathrm{th}}-f_{\mathrm{r} k}^{\mathrm{th}} f_{\mathrm{r} l}^{\mathrm{th}}\right\}, \\
& \gamma_{f_{\mathrm{r}} f_{\mathrm{t}}, k l} \equiv \varepsilon \int_{-\infty}^{0} \mathrm{~d} t\left\{\left(f_{\mathrm{r} k}(0) f_{\mathrm{t} l}(t)\right)^{\mathrm{th}}-f_{\mathrm{r} k}^{\mathrm{th}} f_{\mathrm{t} l}^{\mathrm{th}}\right\} \\
& \gamma_{f_{\mathrm{t}} f_{\mathrm{r}}, k l} \equiv \varepsilon \int_{-\infty}^{0} \mathrm{~d} t\left\{\left(f_{\mathrm{t} k}(0) f_{\mathrm{rl} l}(t)\right)^{\mathrm{th}}-f_{\mathrm{t} k}^{\mathrm{th}} f_{\mathrm{r} l}^{\mathrm{th}}\right\} \\
& \gamma_{f_{\mathrm{t}} f_{\mathrm{t}}, k l} \equiv \varepsilon \int_{-\infty}^{0} \mathrm{~d} t\left\{\left(f_{\mathrm{t} k}(0) f_{\mathrm{t} l}(t)\right)^{\mathrm{th}}-f_{\mathrm{t} k}^{\mathrm{th}} f_{\mathrm{t} l}^{\mathrm{th}}\right\}, \quad k, l=1,2,3 .
\end{aligned}
$$

With this and the assumed long-time behaviour of the correlation functions, the $\gamma^{\prime}$ s are $O\left(\varepsilon^{0}\right)$. 


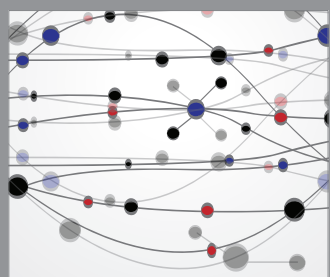

The Scientific World Journal


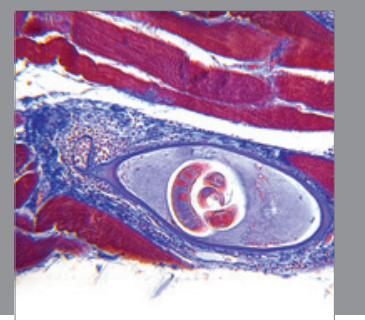

Gastroenterology

Research and Practice
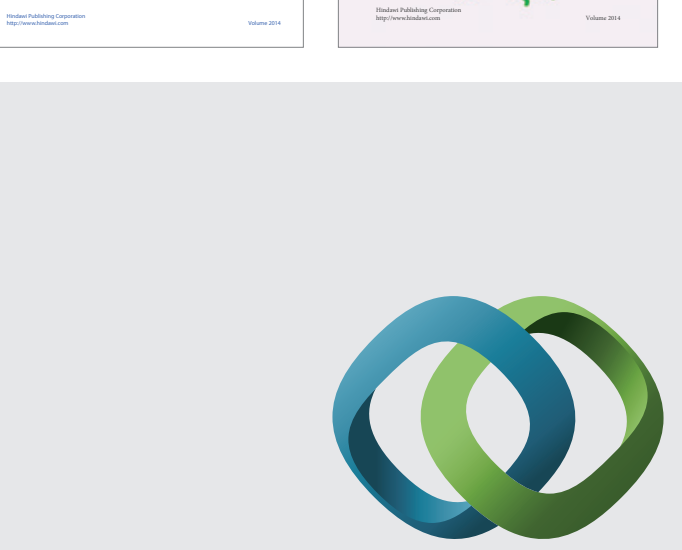

\section{Hindawi}

Submit your manuscripts at

http://www.hindawi.com
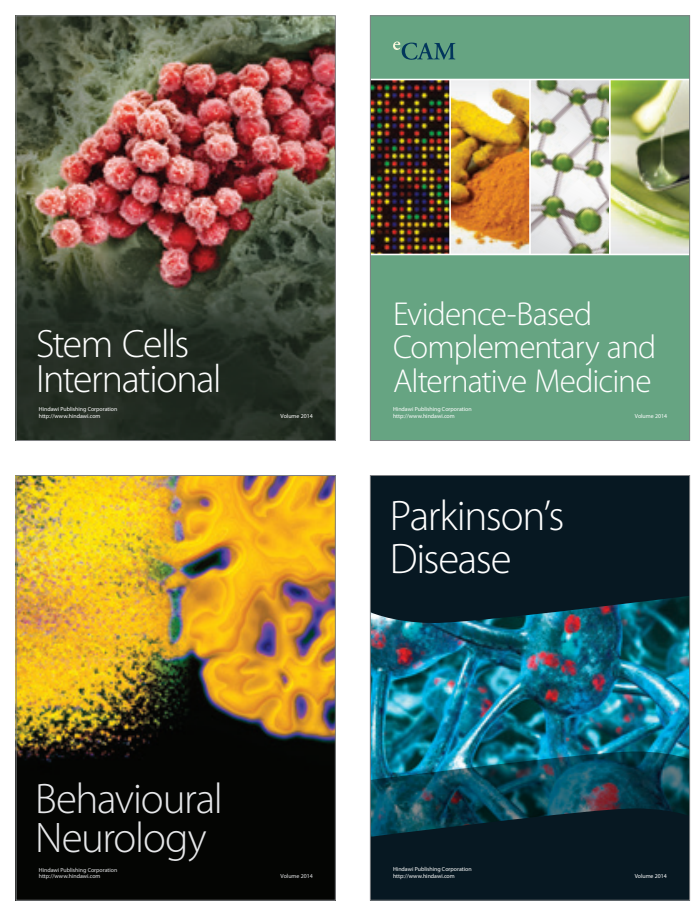

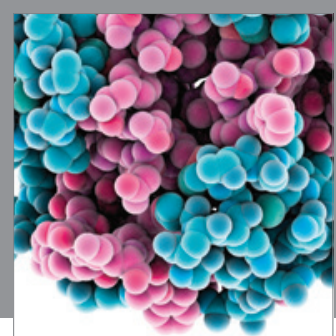

Journal of
Diabetes Research



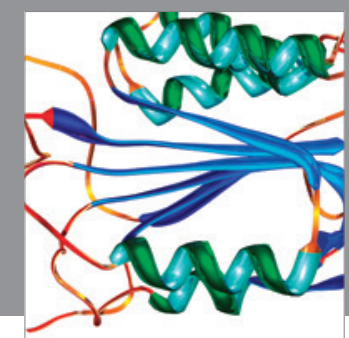

Disease Markers
